This is the author's version of the accepted, peer-reviewed chapter:

Zlatar M., Schläpfer CW., Daul C. (2009) A New Method to Describe the Multimode Jahn-Teller Effect Using Density Functional Theory. In: Köppel H., Yarkony D., Barentzen H. (eds) The Jahn-Teller Effect. Springer Series in Chemical Physics, vol 97. Springer, Berlin, Heidelberg. DOI: https://doi.org/10.1007/978-3-642-03432-9_6

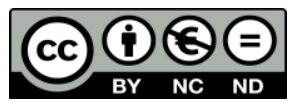

This work is licensed under the Attribution-NonCommercial-NoDerivatives 4.0 International (CC BY-NC-ND 4.0) 


\title{
A New Method to Describe the Multimode Jahn-Teller Effect Using Density Functional Theory
}

\author{
Matija Zlatar, Carl-Wilhelm Schläpfer, and Claude Daul \\ Department of Chemistry, University of Fribourg, Switzerland \\ Center for Chemistry, IHTM, University of Belgrade, Serbia
}

\begin{abstract}
A new method for the analysis of the adiabatic potential energy surfaces of Jahn-Teller (JT) active molecules is presented. It is based on the analogy between the JT distortion and reaction coordinates. Within the harmonic approximation the JT distortion can be analysed as the linear combination of all totally symmetric normal modes in the low symmetry minimum energy conformation. Contribution of the normal modes to the distortion, their energy contribution to the JT stabilisation energy, the forces at high symmetry cusp and detailed distortion path can be estimated quantitatively. This approach gives direct insight into the coupling of electronic structure and nuclear displacements. Further more, it is reviewed how multideterminental DFT can be applied for the calculation of the JT parameters. As examples the results for $\mathrm{VCl}_{4}$, cyclopentadienyl radical and cobaltocene are given.
\end{abstract}

\section{Introduction}

The Jahn-Teller (JT) theorem states that a molecule with a degenerate electronic ground state spontaneously distorts along non-totally symmetric vibrational coordinates. This removes the degeneracy and lowers the energy. At the point of electronic degeneracy the Born-Oppenheimer (BO) [18], or adiabatic, approximation breaks down and there is vibronic coupling between electronic states and nuclear motion. The theory underlying the JT and related effects, is well known and documented in detail [15]. It is based on a perturbation expression of the potential energy surface near the point of electronic degeneracy. The coefficients in the expression of potential energy are called vibronic coupling coefficients, and they have a physical meaning. One of the goals in the analysis of JT systems is the determination of these parameters, and rationalizing the microscopic origin of the problem.

Despite the big advance in various experimental techniques used to study the JT effect, it is not sufficient to understand the latter based only on experimental data. Computational methods are, thus, necessary to get deeper insight into the system under study and to predict the properties of unknown ones. Traditional first principles methods can still be used even where non-adiabatic effects are important, if the BO approximation is reintroduced by the perturbation approach. Density 
Functional Theory (DFT) is the most common theoretical method in quantum chemistry today, but there are still erroneous beliefs that it is not able to handle degenerate states. E.g. Bersuker [14] and Kaplan [49] emphasised that DFT techniques are not adequate to reproduce vibronic effects. In contrary, DFT can be applied to both, degenerate and excited states, as formally proved by the reformulation of the original Hohenberg-Kohn theorems - constrained search method and finite temperature DFT [63]. Further more, Kohn-Sham (KS) DFT, as the most common practical way of using DFT, is based on the equations equivalent and fully compatible with equations used in wave-function based methods. Conventional single determinant DFT has been extended to handle the multiplet problem [27,84]. A method based on this multideterminental DFT, for the study of the JT systems was developed in our group [21] and reviewed in this article. The theory behind the DFT is well elaborated and will not be presented in this review. The reader interested in this subject is referred to a several good and comprehensive reviews or books e.g. [31, 51,63] and to the references therein.

The JT effect is dictated by the molecular symmetry. Group theory allows identifying the symmetry of the JT distortion, which is for simple molecules usually determined by one single normal coordinate that satisfies the symmetry requirements. In complex molecules, the JT distortion is a superposition of many different normal coordinates. In the JT semantics this is called the multimode problem. In this review the treatment of this problem using DFT recently proposed by us [86] is presented. The essence of our proposition is to express JT active distortion as a linear combination of all totally symmetric normal modes in the low symmetry minimum energy conformation. It is based on the fact that JT distortion is analogous to a reaction coordinate. The reaction coordinate belongs to the totally symmetric irreducible representation of the molecular point group of the energy minimum conformation, as proved by Bader [9-11] and Pearson [64, 65]. This is so even if a complicated nuclear motion is considered for the reaction coordinate. The JT distortion can always be written as a sum of totally symmetric normal modes. A detailed analysis of the different contributions of the normal modes is of interest, because it gives direct insight into the coupling of electronic structure and nuclear movements. This is of a particular interest in various fields of chemistry, e.g. in coordination, bioinorganic, material chemistry, or in discussing reaction mechanisms.

This review is organized in the following way. In Sect. 2 the vibronic coupling theory used in this work will be presented, with an emphasise on the different aspects and meaning of vibronic coupling constants. Several simple examples are given to show how the group theory is used for a qualitative discussion. In Sect. 3 we show how DFT can be applied for the calculation of the JT parameters. Section 4 contains some particular examples from our work as illustration of the concepts discussed in Sects. 2 and 3. They are tetrachlorovanadium(IV) $\left(\mathrm{VCl}_{4}\right)$ in 4.1 , cyclopentadienyl radical $\left(\mathrm{C}_{5} \mathrm{H}_{5}\right)$ in 4.2 , and cobaltocene $\left(\mathrm{CoCp}_{2}\right)$ in 4.3 . In the Sect. 5 our model for the analysis of the multimode JT effect is described in detail. Finally, conclusions and perspectives are given in Sect. 6. In Sect. 7 computational details are reported. 


\section{Relevant Theory of JT Effect}

Vibronic coupling theory has been applied to explain Electron Paramagnetic Resonance (EPR), Raman and UV/VIS spectra of some JT-active molecules. Model Hamiltonians were used to fit to the experiments. Some of the early work on various aspects of the vibronic coupling was done by e.g. van Vleck [77], Öpyk and Pryce [62], Longuet-Higgins [25, 56, 57], Liehr [55, 60], Herzberg [41, 42], etc. For the historical development, details about vibronic coupling theory and various application until year 2006 reader is referred to the book by I. B. Bersuker [46] and to the references therein. We would like to emphasize the works of Bader [9-11] and Pearson $[64,65]$ on the symmetry of reaction coordinates in addition, because it is crucial in our discussion of the multimode problem, as shown in our recent paper [86] and in Sect. 5 of this review.

Consider a $\mathrm{N}$-atomic molecule in the high-symmetry (HS) nuclear configuration, $\mathbf{R}_{\mathrm{HS}}$, in point-group $G_{\mathrm{HS}} . \mathcal{H}^{\mathrm{HS}}$ is the electronic Hamilton operator, which defines the electronic structure. The molecule has $3 N-6$ normal coordinates $\mathbf{Q}_{\mathrm{HSk}}, k=$ $1, \ldots, 3 N-6\left(3 N-5\right.$ in the case of linear molecules), ${ }^{1}$ which can be classified according to the corresponding irreps, $\Gamma_{\mathrm{HSk}}^{v i b}$, of the point-group $G_{\mathrm{HS}}{ }^{2} \mathrm{In}$ order to discuss the potential energy surface, the electronic Hamiltonian, $\mathcal{H}$, is expanded as a Taylor series around the HS point $\mathbf{R}_{\mathrm{HS}}$, along the orthonormal $\mathbf{Q}_{\mathrm{HSk}}$ :

$$
\begin{gathered}
\mathcal{H}=\mathcal{H}^{\mathrm{HS}}+\sum_{\mathrm{k}=1}^{3 N-6}\left(\frac{\partial V}{\partial \mathbf{Q}_{\mathrm{HSk}}}\right)_{\mathrm{HS}} \mathbf{Q}_{\mathrm{HSk}}+\frac{1}{2} \sum_{\mathrm{k}, \mathrm{l}=1}^{3 N-6}\left(\frac{\partial^{2} V}{\partial \mathbf{Q}_{\mathrm{HSk}} \mathbf{Q}_{\mathrm{HSl}}}\right)_{\mathrm{HS}} \mathbf{Q}_{\mathrm{HSk}} \mathbf{Q}_{\mathrm{HSl}}+\ldots \\
\mathcal{H}=\mathcal{H}^{\mathrm{HS}}+\mathcal{W} .
\end{gathered}
$$

$\mathcal{W}$ represents vibronic operator (JT Hamiltonian) and is a perturbation on the $\mathcal{H}^{\mathrm{HS}}$.

Next, consider that the ground state eigenfunction of $\mathcal{H}^{\mathrm{HS}}$ with energy, $E^{0}$, is f-fold degenerate, $\Psi_{\mathrm{i}}^{\mathrm{HS}, 0}=\left|\Gamma_{\mathrm{HS}}^{\text {elect }} m_{\mathrm{i}}\right\rangle . \Gamma_{\mathrm{HS}}^{\text {elect }}$ is irrep of the ground state and $m_{\mathrm{i}}$ the component, $\mathrm{i}=1, \ldots, \mathrm{f}$. This leads to an $\mathrm{f}$-fold $\mathrm{JT}$ effect. The matrix elements, $H_{\mathrm{ij}}$, of $\mathcal{H}$ within the basis functions $\Psi_{\mathrm{i}}^{\mathrm{HS}, 0}$, are given, according to the conventional second-order perturbation theory, where 0 designate the ground state, and $\mathrm{p}$ excited states:

\footnotetext{
${ }^{1}$ As it will be described in Sect. 5 our analysis of the multimode JT effect is based on the normalcoordinate analysis from the low symmetry points, contrary to the conventional vibronic-coupling theory. Therefore we distinguish between the normal coordinates in the HS conformation, $\mathbf{Q}_{\mathrm{HSk}}$, and the normal coordinates in the stable low symmetry (LS) conformation $\mathbf{Q}_{\mathrm{k}}$.

${ }^{2}$ In general discussions label of the irreducible representation is $\Gamma$. To differentiate between the symmetry of electronic states and vibrations, irreps of point groups $G_{\mathrm{HS}}$ and $G_{\mathrm{LS}}$ we add subscript and superscript, e.g. $\Gamma_{\mathrm{HS}}^{\text {elect }}, \Gamma_{\mathrm{HS}}^{v i b}$ etc. In particular examples Mulliken symbols are used, e.g. $A_{1}$, $B_{2}$ etc. Electronic states are labelled with upper-case letters, e.g. ${ }^{2} E$, while one-electron orbitals with lower-case, e.g. configuration $e^{0.5} e^{0.5}$. Symmetry of the normal modes are denoted also with lower case letters, e.g. $a_{1}$ vibration, or in general as $\gamma$.
} 


$$
H_{\mathrm{ij}}=E^{0} \delta_{\mathrm{ij}}+\sum_{\mathrm{i}, \mathrm{j}=1}^{\mathrm{f}}\left\langle\Psi_{\mathrm{i}}^{\mathrm{HS}, 0}|\mathcal{W}| \Psi_{\mathrm{j}}^{\mathrm{HS}, 0}\right\rangle+\sum_{\mathrm{i}=1}^{\mathrm{f}} \sum_{\mathrm{p} \neq 0} \frac{\left|\left\langle\Psi_{\mathrm{i}}^{\mathrm{HS}, 0}|\mathcal{W}| \Psi^{\mathrm{HS}, \mathrm{p}}\right\rangle\right|^{2}}{E^{0}-E^{\mathrm{p}}}+\ldots
$$

This formulation defines potential energy surface around $\mathbf{R}_{\mathrm{HS}}$ (or in general around any point $\mathbf{R}_{\mathrm{X}}$ ) and allows a discussion of the Jahn-Teller (JT) effect [47], the pseudo-Jahn-Teller (PJT) effect [9-11,46,64,65], the Renner-Teller (RT) effect $[46,66]$ as well as the chemical reactivity $[9-11,64,65]$, with the same formalism [46]. Keeping the terms up to second order in $\mathbf{Q}_{\mathrm{HSk}}$ :

$$
\begin{aligned}
& H_{\mathrm{ij}}=E^{0} \delta_{\mathrm{ij}}+\sum_{\mathrm{k}=1}^{3 N-6} \sum_{\mathrm{i}, \mathrm{j}=1}^{\mathrm{f}}\langle\underbrace{\left\langle\Psi_{\mathrm{i}}^{\mathrm{HS}, 0}\left|\left(\frac{\partial V}{\partial \mathbf{Q}_{\mathrm{HSk}}}\right)_{H S}\right| \Psi_{\mathrm{j}}^{\mathrm{HS}, 0}\right\rangle}_{F_{\mathrm{ij}}^{\mathrm{k}}} \mathbf{Q}_{\mathrm{HSk}} \\
& +\frac{1}{2} \sum_{\mathrm{k}=1}^{3 N-6} \sum_{\mathrm{i}=1}^{\mathrm{f}}\langle\underbrace{\left\langle\Psi_{\mathrm{i}}^{\mathrm{HS}, 0}\left|\left(\frac{\partial^{2} V}{\partial \mathbf{Q}_{\mathrm{HSk}}^{2}}\right)_{\mathrm{HS}}\right| \Psi_{\mathrm{i}}^{\mathrm{HS}, 0}\right\rangle}_{K_{0}} \mathbf{Q}_{\mathrm{HSk}}^{2} \\
& +\frac{1}{2} \sum_{\mathrm{k}, \mathrm{l}=1 ; \mathrm{k} \neq 1 \mathrm{i}, \mathrm{j}=1 ; \mathrm{i} \neq \mathrm{j}}^{3 N-6} \underbrace{\mathrm{f}}_{G_{\mathrm{ij}}^{\mathrm{kl}}} \underbrace{\left.\Psi_{\mathrm{HS}, 0}\left|\left(\frac{\partial^{2} V}{\partial \mathbf{Q}_{\mathrm{HSk}} \mathbf{Q}_{\mathrm{HSl}}}\right)_{H S}\right| \Psi_{\mathrm{j}}^{\mathrm{HS}, 0}\right\rangle}_{\mathrm{i}} \mathbf{Q}_{\mathrm{HSk}} \mathbf{Q}_{\mathrm{HS}} \\
& +\sum_{\mathrm{k}=1}^{3 N-6} \sum_{\mathrm{i}=1}^{\mathrm{f}} \sum_{\mathrm{p} \neq 0} \underbrace{\frac{\left|\left\langle\Psi_{\mathrm{i}}^{\mathrm{HS}, 0}\left|\left(\frac{\partial V}{\partial \mathbf{Q}_{\mathrm{HSk}}}\right)_{H S}\right| \Psi^{\mathrm{HS}, \mathrm{p}}\right\rangle\right|^{2}}{E^{0}-E^{\mathrm{p}}}}_{R_{\mathrm{ip}}} \mathbf{Q}_{\mathrm{HSk}}^{2} .
\end{aligned}
$$

The matrix elements in (4) are vibronic coupling constants, thus (4) can be rewritten as:

$$
\begin{aligned}
H_{\mathrm{ij}}= & E^{0} \delta_{\mathrm{ij}}+\sum_{\mathrm{k}=1}^{3 N-6} \sum_{\mathrm{i}, \mathrm{j}=1}^{\mathrm{f}} F_{\mathrm{ij}}^{\mathrm{k}} \mathbf{Q}_{\mathrm{HSk}}+\frac{1}{2} \sum_{\mathrm{k}=1}^{3 N-6} \sum_{\mathrm{i}=1}^{\mathrm{f}} K_{0} \mathbf{Q}_{\mathrm{HSk}}^{2} \\
& +\frac{1}{2} \sum_{\mathrm{k}, \mathrm{l}=1 ; \mathrm{k} \neq 1 \mathrm{i}, \mathrm{j}=1 ; \mathrm{i} \neq \mathrm{j}}^{3 N-6} G_{\mathrm{ij}}^{\mathrm{kl}} \mathbf{Q}_{\mathrm{HSk}} \mathbf{Q}_{\mathrm{HSl}}+\sum_{\mathrm{k}=1}^{3 N-6} \sum_{\mathrm{i}=1}^{\mathrm{f}} \sum_{\mathrm{p} \neq 0} R_{\mathrm{ip}} \mathbf{Q}_{\mathrm{HSk}}^{2}
\end{aligned}
$$

The definition of the vibronic coupling constants is given in (6), (7), (8) and (9):

The terms

$$
F_{\mathrm{ij}}^{\mathrm{k}}=\left\langle\Psi_{\mathrm{i}}^{\mathrm{HS}, 0}\left|\left(\frac{\partial V}{\partial \mathbf{Q}_{\mathrm{HSk}}}\right)_{H S}\right| \Psi_{\mathrm{j}}^{\mathrm{HS}, 0}\right\rangle
$$


are the linear vibronic coupling constants;

$$
\text { The terms } \quad G_{\mathrm{ij}}^{\mathrm{kl}}=\left\langle\Psi_{\mathrm{i}}^{\mathrm{HS}, 0}\left|\left(\frac{\partial^{2} V}{\partial \mathbf{Q}_{\mathrm{HSk}} \mathbf{Q}_{\mathrm{HSl}}}\right)_{H S}\right| \Psi_{\mathrm{j}}^{\mathrm{HS}, 0}\right\rangle
$$

are the quadratic vibronic coupling constants;

$$
\text { The terms } \quad G_{\mathrm{ii}}^{\mathrm{kk}}=K_{0}=\left\langle\Psi_{\mathrm{i}}^{\mathrm{HS}, 0}\left|\left(\frac{\partial^{2} V}{\partial \mathbf{Q}_{\mathrm{HSk}}^{2}}\right)_{\mathrm{HS}}\right| \Psi_{\mathrm{i}}^{\mathrm{HS}, 0}\right\rangle
$$

are the harmonic force constants at HS point;

$$
\text { The terms } \quad R_{\text {ip }}=\frac{1}{2} K_{v}=\frac{\left|\left\langle\Psi_{\mathrm{i}}^{\mathrm{HS}, 0}\left|\left(\frac{\partial V}{\partial \mathbf{Q}_{\mathrm{HSk}}}\right)_{H S}\right| \Psi^{\mathrm{HS}, \mathrm{p}}\right\rangle\right|^{2}}{E^{0}-E^{\mathrm{p}}}
$$

are the electronic relaxation.

The complexity of (4) is reduced by symmetry rules, which allow identifying the non zero vibronic coupling constants. The Hamiltonian is invariant under all symmetry operations of the corresponding point group. Therefore, the operator $\partial V / \partial \mathbf{Q}_{\mathrm{HSk}}$ is transforming according to the irreducible representation $\Gamma_{\mathrm{HSk}}^{v i b}$ of the normal coordinate $\mathbf{Q}_{\mathrm{HSk}}$. The operator $\partial^{2} V / \partial \mathbf{Q}_{\mathrm{HSk}} \mathbf{Q}_{\mathrm{HSl}}$ represents a basis for the reducible representation obtained by direct product $\Gamma_{\mathrm{HSk}}^{v i b} \otimes \Gamma_{\mathrm{HSl}}^{v i b} \subset \Gamma_{\mathrm{r}} \cdot{ }^{3}$ Hence, the matrix elements in (4) are only different from zero for $\Gamma_{\mathrm{HSk}}^{v i b} \subset \Gamma_{\mathrm{HS}}^{\text {elect }} \otimes \Gamma_{\mathrm{HS}}^{\text {elect }}$ or in the case of the quadratic vibronic coupling constants for $\Gamma_{\mathrm{HSk}}^{v i b} \otimes \Gamma_{\mathrm{HSl}}^{v i b} \subset$ $\Gamma_{\mathrm{HS}}^{\text {elect }} \otimes \Gamma_{\mathrm{HS}}^{\text {elect }}$.

The slope of the potential energy along the direction $\mathbf{Q}_{\mathrm{HSk}}$, is given by the diagonal linear vibronic constant, $F_{\mathrm{ii}}^{\mathrm{k}} . F_{\mathrm{ii}}^{\mathrm{k}}$ represents the force, which moves the nuclei and leads to a change of the structure. These terms are zero at any stationary point on the potential energy surface. If the ground state is nondegenerate the integral will vanish unless $\mathbf{Q}_{\mathrm{HSk}}$ is totally symmetric. Therefore for a system with a nondegenerate ground state, the potential energy surface shows only a gradient along totally symmetric distortions. As a consequence, for any non stationary point, the point group does not change along any reaction path [65]. If the ground state is degenerate, $\mathbf{Q}_{\text {HSk }}$ might be a basis for a non-totally symmetric representation. This is a case if $\mathbf{Q}_{\mathrm{HSk}}$ belongs to one of the irreps which is a component of the direct product $\Gamma_{\mathrm{HS}}^{\text {elect }} \otimes \Gamma_{\mathrm{HS}}^{\text {elect }}$. The spontaneous distortion along these non-totally symmetric normal coordinates, $\mathbf{Q}_{\mathrm{HSk}} \mathrm{s}$, leads to a descent in symmetry and removes the degeneracy of the ground state. When the symmetry is lowered, $\Psi_{\mathrm{i}}^{\mathrm{HS}, 0}$ is no longer degenerate, and the $F_{\text {ii }}^{\mathrm{k}}$ will be zero unless the $\mathbf{Q}_{\mathrm{HSk}}$ becomes totally symmetric in the new point group. The movement of nuclei that were non-totally symmetric in the $G_{\mathrm{HS}}$, must now become totally symmetric. The point group $G_{\mathrm{LS}}$ of the minimum on

\footnotetext{
${ }^{3}$ Totally symmetric component of the direct product $\Gamma_{\mathrm{HSk}}^{v i b} \otimes \Gamma_{\mathrm{HSI}}^{v i b}$ yields the harmonic force constant, $K_{0}$, which is separate term in (4).
} 
the potential energy surface, can be predicted by looking at the correlation tables for the symmetry descent, e.g. in [7]. The point group $G_{\mathrm{LS}}$ is the one in which the mode becomes totally symmetric. If there are several possibilities for a descent in symmetry, $G_{\mathrm{LS}}$ of the minimal energy conformation is the highest one with lifted degeneracy according to the epikernal principle [23, 24, 46]. Jahn and Teller [47] examined all degenerate terms of the symmetry point groups of non-linear molecules, and showed that there is always at least one non-totally symmetric vibration for which the $F_{\mathrm{ij}}^{\mathrm{k}} \neq 0$. This holds even for double groups, in this case $\Psi_{\mathrm{i}}^{\mathrm{HS}, 0}$ is a Kramers doublet. This is the physical basis of the (first order) JT effect. The JT problems are classified according to the symmetry types of the electronic states and the vibrations that are coupled, $\Gamma \otimes \gamma$ [46]. For example, $E \otimes e \mathrm{JT}$ problem denotes, coupling of the degenerate electronic state of irrep $E$, by a degenerate vibration of irrep $e$. Since the slope of the potential surface at the high symmetry configuration, $\mathbf{R}_{\mathrm{HS}}$, is nonzero, this conformation corresponds not to a stationary point. It represents a cusp of the potential energy surface obtained in conventional DFT.

The curvature of the potential energy surface in the direction $\mathbf{Q}_{\mathrm{HSk}}$ at $\mathbf{R}_{\mathrm{HS}}$, is measured by the force constant, $K_{k}=K_{0}+K_{v}$ [46]. The diagonal matrix elements of the second derivative of the potential energy operator, are the primary or nonvibronic force constants, $K_{0}$ [46]. $K_{0}$ is always different from zero and positive $[46,70]$. It represents a restoring force that tends to bring the system back to the more symmetrical situation. HS configuration represents the most stable configuration of the molecule, if the vibronic coupling is ignored, as it minimizes electron-electron repulsion.

The electronic relaxation, $R_{\text {ip }}=\frac{1}{2} K_{v}$, depicts the coupling of the ground state with excited states. This term is always negative, due to the nominator $E^{0}-E^{p}$. Generally it is different from zero, because there is always some excited states, of the same irrep as the ground state. It becomes increasingly important when the ground and the excited states are close in energy. It is referred to as the vibronic force constant, $K_{v}$ [46]. It is responsible for: (1) the negative curvature along the reaction coordinate of the potential energy surface at a transition state [65] (2) for the pseudo-Jahn-Teller effect $[46,65]$, configurational instability of polyatomic species with nondegenerate electronic states; (3) for the avoided crossing between the states of the same symmetry; (4) for the softening of the ground state curvature at the energy minimum conformation; and (5) it contributes to the anharmonicity of the vibrations. In practice, in the analysis of JT systems, this term is usually neglected, or added to the total, observed force constant $K_{k}$.

The quadratic constants, $G_{\mathrm{ij}}^{\mathrm{kl}}$, in non linear molecules influence the shape of the potential energy surfaces. This is true for the higher order terms, e.g. cubic, and terms $R_{\text {ip }}$ (PJT terms) also. Discussion of the various terms contributing to the warping of the potential energy surface can be found in e.g. [39]. For linear molecules the linear vibronic constants are always zero because the non-totally symmetric vibrations are odd and the degenerate states are even. The quadratic terms however are nonzero, and this may lead to instability of the linear configurations in case of a sufficiently strong coupling. This is physical basis of the RT effect $[46,66]$. 
As we see, the complexity of (4) is already reduced by symmetry rules, which allow us to identify the non zero vibronic coupling constants. Moreover, the application of the Wigner-Eckart theorem $[33,81]$ yields a further reduction of the complexity for degenerate irreducible representations.

$$
\left\langle\Psi_{\mathrm{i}}^{\mathrm{HS}, 0}\left|\left(\frac{\partial V}{\partial \mathbf{Q}_{\mathrm{HSk}}}\right)\right| \Psi_{\mathrm{j}}^{\mathrm{HS}, 0}\right\rangle=C\left(\begin{array}{ccc}
\Gamma_{\mathrm{HS}}^{\text {elect }} & \Gamma_{\mathrm{HSk}}^{\text {vib }} & \Gamma_{\mathrm{HS}}^{\text {elect }} \\
m_{\mathrm{i}} & m_{\mathrm{k}} & m_{\mathrm{j}}
\end{array}\right)\left\langle\Psi_{\Gamma_{\mathrm{HS}}^{\text {elect }}}^{\mathrm{HS}, 0}\left\|\left(\frac{\partial V}{\partial \mathbf{Q}_{\Gamma_{\mathrm{HSk}}}}\right)\right\| \Psi_{\Gamma_{\mathrm{HS}}^{\text {elect }}}^{\mathrm{HS}, 0}\right\rangle
$$

where $C\left(\begin{array}{ccc}\Gamma_{\mathrm{HS}}^{e l e c t} & \Gamma_{\mathrm{HSk}}^{\text {vib }} & \Gamma_{\mathrm{HS}}^{\text {elect }} \\ m_{\mathrm{i}} & m_{\mathrm{k}} & m_{\mathrm{j}}\end{array}\right)$ are the coupling coefficients of the point group of the molecule at the high symmetry point, $G_{\mathrm{HS}}$, and $\left\langle\Psi_{\Gamma_{\mathrm{HS}}^{\mathrm{HS}, 0}}^{\mathrm{HS}}\left\|\left(\frac{\partial V}{\partial \mathbf{Q}_{\Gamma_{\mathrm{HSk}}}}\right)\right\| \Psi_{\Gamma_{\mathrm{HS}}^{\text {elect }}}^{\mathrm{HS}, 0}\right\rangle$ is reduced matrix-element that only depends upon irreps and not upon their components.

In the case of the quadratic matrix elements, $G_{\mathrm{ij}}^{\mathrm{kl}}$, the Wigner-Eckart theorem $[33,81]$ might be applied similarly to the previous case, where the summations run over all $\Gamma_{\mathrm{r}}$ and their components $m_{\mathrm{r}}$ :

$$
\begin{aligned}
\left\langle\Psi_{\mathrm{i}}^{\mathrm{HS}, 0}\left|\left(\frac{\partial V^{2}}{\partial \mathbf{Q}_{\mathrm{HSk}} \partial \mathbf{Q}_{\mathrm{HSl}}}\right)\right| \Psi_{\mathrm{j}}^{\mathrm{HS}, 0}\right\rangle= & \left\langle\Psi_{\Gamma_{\mathrm{HS}}^{\text {elect }}}^{\mathrm{HS}, 0} \|\left(\frac{\partial V^{2}}{\partial \mathbf{Q}_{\Gamma_{\mathrm{HSk}}} \partial \mathbf{Q}_{\Gamma_{\mathrm{HSl}}}}\right)|| \Psi_{\Gamma_{\mathrm{HS}}^{\text {elect }}}^{\mathrm{HS}, 0}\right\rangle \\
& \sum_{\Gamma_{\mathrm{r}}, m_{\mathrm{r}}} C\left(\begin{array}{cccc}
\Gamma_{\mathrm{HS}}^{\text {elect }} & \Gamma_{\mathrm{r}} & \Gamma_{\mathrm{HS}}^{\text {elect }} \\
m_{\mathrm{i}} & m_{\mathrm{r}} & m_{\mathrm{j}}
\end{array}\right) C\left(\begin{array}{cccc}
\Gamma_{\mathrm{HSk}}^{\text {vib }} & \Gamma_{\mathrm{r}} & \Gamma_{\mathrm{HSl}}^{\text {vib }} \\
m_{\mathrm{k}} & m_{\mathrm{r}} & m_{1}
\end{array}\right) .
\end{aligned}
$$

Thus, only one reduced matrix element has to be calculated or determined experimentally, because the coupling coefficients are known. This simplifies the interpretation considerably. E.g. in the case of a $E \otimes e \mathrm{JT}$ problem the potential energy surface is determined by only three reduced matrix elements, corresponding to the parameters $F, G, K[46]$.

In order to show how the theory given above can be applied, few simple examples are shown. Numerical results obtained from the DFT calculation on these systems are given later, Sect. 4.

The ground electronic state of eclipsed cobaltocene $\left(\mathrm{CoCp}_{2}\right)$ or cyclopentadienyl radical $\left(\mathrm{C}_{5} \mathrm{H}_{5}\right)$, with $D_{5 h}$ symmetry is ${ }^{2} E_{1}^{\prime \prime}$, with a single electron (hole) in the doubly degenerate orbital, $e_{1}^{\prime \prime}$. Using group theory it is easy to show that the distortion coordinate is $e_{2}^{\prime}\left(E_{1}^{\prime \prime} \otimes E_{1}^{\prime \prime} \subset A_{1}^{\prime}+\left[A_{2}^{\prime}\right]+E_{2}^{\prime}\right)$, and the descent in symmetry goes to $C_{2 v}$. The electronic state will split into $A_{2}$ and $B_{1}$, while the degenerate $\mathrm{JT}$ active distortion $e_{2}^{\prime}$ splits into $a_{1}$ and $b_{2}$. Let us analyse the problem in the space of the two components $Q_{a}$ and $Q_{b}$ of $e_{2}^{\prime}\left(Q_{a}\right.$ is of $a_{1}$ symmetry in $C_{2 v}$, and $Q_{b}$ is of $b_{2}$ symmetry). The JT active distortion is the totally symmetric reaction coordinate, $a_{1}$, in $C_{2 v}$. The modes of $b_{2}$ symmetry allow mixing of the two electronic states emerging from the degenerate ground state. The second order vibronic coupling constant, $G_{\mathrm{ij}}^{\mathrm{kl}}$ is zero, because in the direct product $E_{2}^{\prime} \otimes E_{2}^{\prime} \subset A_{1}^{\prime}+\left[A_{2}^{\prime}\right]+E_{1}^{\prime}$ there are no 
Table 1 Coupling coefficients for the $D_{5}$ group

\begin{tabular}{llcccc}
\hline & el.state & \multicolumn{5}{c}{$E_{1} \times E_{1}$} \\
vib. & component & $A A$ & $A B$ & $B A$ & $B B$ \\
\hline$e_{2}$ & $b$ & 0 & $-\frac{1}{\sqrt{2}}$ & $-\frac{1}{\sqrt{2}}$ & 0 \\
& $a$ & $\frac{1}{\sqrt{2}}$ & 0 & 0 & $-\frac{1}{\sqrt{2}}$ \\
\hline
\end{tabular}

terms of $E_{2}^{\prime}$ symmetry able to interact with the $E_{1}^{\prime \prime}$ electronic wavefunctions. There is no warping of the Mexican hat. The totally symmetric component of $E_{2}^{\prime} \otimes E_{2}^{\prime}$ representation yields the harmonic force field constant, $K$. Using Wigner-Eckart theorem $[33,81]$ and the coupling coefficients for the $D_{5 h}$ point group, Table 1 . it is easy to see that the following integrals vanish:

$$
\left\langle\Psi_{A}\left|\frac{\partial V}{\partial \mathbf{Q}_{a}}\right| \Psi_{B}\right\rangle=\left\langle\Psi_{B}\left|\frac{\partial V}{\partial \mathbf{Q}_{a}}\right| \Psi_{A}\right\rangle=\left\langle\Psi_{A}\left|\frac{\partial V}{\partial \mathbf{Q}_{b}}\right| \Psi_{A}\right\rangle=\left\langle\Psi_{B}\left|\frac{\partial V}{\partial \mathbf{Q}_{b}}\right| \Psi_{B}\right\rangle=0
$$

and the remaining integrals are:

$$
\begin{aligned}
F=\left\langle\Psi_{A}\left|\frac{\partial V}{\partial \mathbf{Q}_{a}}\right| \Psi_{A}\right\rangle & =-\left\langle\Psi_{B}\left|\frac{\partial V}{\partial \mathbf{Q}_{a}}\right| \Psi_{B}\right\rangle=-\left\langle\Psi_{A}\left|\frac{\partial V}{\partial \mathbf{Q}_{b}}\right| \Psi_{B}\right\rangle=-\left\langle\Psi_{B}\left|\frac{\partial V}{\partial \mathbf{Q}_{b}}\right| \Psi_{A}\right\rangle \\
& =\frac{1}{\sqrt{2}}\left\langle\Psi_{E_{1}^{\prime \prime}} \| \frac{\partial V}{\partial \mathbf{Q}_{E_{2}^{\prime}}}|| \Psi_{E_{1}^{\prime \prime}}\right\rangle .
\end{aligned}
$$

Potential energy as a function of a distortion along $\mathbf{Q}_{a}$ and $\mathbf{Q}_{\mathbf{b}}$ is:

$$
E=E^{0}+\frac{1}{2} K\left(\mathbf{Q}_{\mathbf{a}}{ }^{2}+\mathbf{Q}_{\mathbf{b}}{ }^{2}\right) \pm F\left[\left(\mathbf{Q}_{\mathbf{a}}{ }^{2}+\mathbf{Q}_{\mathbf{b}}{ }^{2}\right)\right]^{\frac{1}{2}} .
$$

The energy change along $\mathbf{Q}_{a}$, or $\mathbf{Q}_{b}$ or along any linear combination is the same. In this expression only quadratic forms of $\mathbf{Q}_{a}$ and $\mathbf{Q}_{b}$ are present, thus the energy of a distortion along $-\mathbf{Q}_{a}$ is the same energy as along $\mathbf{Q}_{a}$, thus only the other component of the degenerate state is stabilized. The potential energy surface has a Mexican-hat shape, without any warping. Energy change is the same in all directions in the two dimensional space spanned by these two coordinates.

In the case of $\mathrm{CoCp}_{2}$ there are six different $e_{2}^{\prime}$ modes and in the case of $\mathrm{C}_{5} \mathrm{H}_{5}$ four and each of them will be characterized with one pair of parameters $F$ and $K$.

The quadratic vibronic constants of the $e_{1}^{\prime}$ normal coordinates are not zero even if the linear ones are zero because in the $D_{5 h}$ point group $E_{1}^{\prime} \otimes E_{1}^{\prime} \subset A_{1}^{\prime}+\left[A_{2}^{\prime}\right]+$ $E_{2}^{\prime}$. In HS, thus the first order and the second order JT effect are separated. As one component of $e_{1}^{\prime}$ becomes in LS totally symmetric too, they will also mix and contribute to the totally symmetric JT coordinate. Thus, we see that considering only one normal coordinate is not enough to describe the JT effect even in this simple case. In the subsequent sections we will address this problem again, and propose how to analyse which is the contribution of the different vibrations to the total distortion of a molecule, and which of them are the most important driving force for the distortion. 
Next, let see how group theory can be used in determining the symmetry properties of the JT distortions in a tetrahedral, $T_{d}$, molecule with an $E$ ground electronic state, e.g. $\mathrm{VCl}_{4}$, Sect. 4.1. The symmetry of the $\mathrm{JT}$ active vibration is determined as $E \otimes E \subset A_{1}+\left[A_{2}\right]+E$. This is another example of an $E \otimes e \mathrm{JT}$ problem. Symmetry lowering is $T_{d} \rightarrow D_{2 d}$. In lower symmetry $e$ vibration splits into $a_{1}+b_{1}$. Only one component of the degenerate vibration is JT active. JT distortion is along the totally symmetric reaction coordinate, $a_{1}$, in $D_{2 d}$. The potential along the direction of the JT inactive vibrations is parabolic with a minimum for the high symmetry conformation. In this case quadratic vibronic coupling constant is different from zero, as there is always $E$ terms present in the direct product of $E \otimes E$, and the potential energy surface has a famous Mexican-hat-like form, with three equivalent minima and three equivalent transition states. The distortion along $+\mathbf{Q}_{a_{1}}$ and $-\mathbf{Q}_{a_{1}}$ are not identical. The energy of the two different states is not the same.

\section{DFT Calculation of the JT Ground State Properties}

As seen in the Sect. 2, JT effect is governed by the symmetry properties of $G_{\mathrm{HS}}$ and $G_{\mathrm{LS}}$ point groups of the studied molecule. The information from group theory can be used for a qualitative discussion. This does not tell anything about the degree of the distortion or how big the energy gain is due to the descent in symmetry. These questions are of fundamental importance to characterize JT systems. To answer them it is necessary either to perform the experiment, and fit the results to the proposed model, or to carry out a computational study.

The vibronic coupling constants discussed in Sect. 2 define the potential energy surface. A qualitative cut through the potential energy surface, along JT active vibration $\mathbf{Q}_{a}$ is given in Fig. 1. The figure indicates how the parameters $E_{\mathrm{JT}}$ (the JT stabilization energy), $\Delta$ (the warping barrier), $R_{\mathrm{JT}}$ (the $\mathrm{JT}$ radius) and $E_{\mathrm{FC}}$ (the

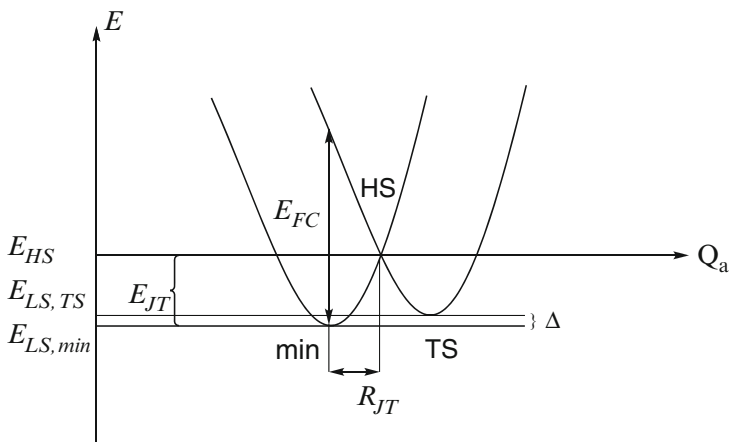

Fig. 1 Qualitative cross section through the potential energy surface, along JT active vibration $\mathbf{Q}_{a}$; Definition of the JT parameters - the JT stabilisation energy, $E_{\mathrm{JT}}$, the warping barrier, $\Delta$, the $\mathrm{JT}$ radius, $R_{\mathrm{JT}}$, the energy of the vertical Frank-Condon transition, $E_{\mathrm{FC}}$ 
Frank-Condon transition) define the potential energy surface. The meaning of the parameters is clear - energy stabilization due to the JT effect is given by the value of $E_{\mathrm{JT}}$ (or alternatively by $E_{\mathrm{FC}}=4 E_{\mathrm{JT}}$ ), and direction and magnitude of the distortion by the $R_{\mathrm{JT}}$.

Using non-empirical methods it is, at least in principle, easy to calculate this alternative set of parameters. They are connected to the set of parameters discussed in previous Sect. 2, e.g. for the $E \otimes e$ JT problem using following expressions, (15), (16), (17):

$$
\begin{gathered}
E_{\mathrm{JT}}=\frac{F^{2}}{2(K-2|G|)} . \\
\Delta=\frac{4 E_{\mathrm{JT}}|G|}{K+2|G|}, \\
R_{\mathrm{JT}}^{\min }=\frac{|F|}{K-2|G|} \quad R_{\mathrm{JT}}^{\mathrm{TS}}=-\frac{|F|}{K+2|G|},
\end{gathered}
$$

Similar expressions, for other type of JT problems can be found in [46].

DFT is the modern alternative to the wave-function based ab initio methods and allows to obtain accurate results at low computational cost, that also helps to understand the chemical origin of the effect. DFT, like Hartree-Fock (HF) methods, exploit molecular symmetry which is crucial in the case of computational studies of the JT effect. It also includes correlation effects into the Hamiltonian via the exchange-correlation functional. HF and many-body perturbation methods are found to perform poorly in the analysis of JT systems for obvious reasons, at contrast to the methods based on DFT, or multiconfigurational SCF and coupled cluster based methods [73]. The later are very accurate but have some drawbacks, mainly the very high computational cost that limits the applications to the smaller systems only. Another drawback is the choice of the active space which involves arbitrariness.

In order to get the JT parameters, it is necessary to know geometries and energies of HS and LS points. For the LS points, as they are in non-degenerate electronic ground state, at least formally, this is straightforward. Electronic structure of the HS point, on the other hand, must be represented with at least two Slater determinants, consequently, using a single determinant DFT is troublesome. Wang and Shwarz [79], or Baerends [69] pointed out that a single determinant KS-DFT is deficient in the description of (near) degeneracy correlation. In a non-empirical approach to calculate the JT distortion using DFT [21] it was proposed to use average of configuration (AOC) calculation to generate the electron density. This is a SCF calculation where the electrons of degenerate orbitals are distributed equally over the components of the degenerate irreps leading to a homogeneous distribution of electrons with partial occupation, in order to retain the $A_{1}$ symmetry of the total density in the HS point group. E.g. for $e^{1}$ configuration this will mean to place 0.5 electrons into each of the two $e$ orbitals. This calculation yields the geometry of the high symmetry species. 
The idea of fractional occupation numbers was introduced by Slater [71], already in 1969. This approach is not limited for the JT systems, e.g. it was explored by Dunlap and Mei [32] for molecules, by Filatov and Shaik [36] for diradicals, and is also used for calculations of solids and metal clusters [8]. It rests on a firm basis in cases when the ground state density has to be represented by a weighted sum of single determinant densities $[53,79]$. One should remember that molecular orbitals (MO) themselves have no special meaning. Thus, using partial occupation is just a way of obtainning electron density of a proper symmetry (HS).

Although, AOC calculation gives us geometry of a HS point, using simply the energy obtained in this way would be erroneous. AOC calculation is giving too low energy. The JT stabilization energy is not simply the energy difference between the HS and the LS species. This is due to the self interaction error (SIE) present in the approximate exchange-correlation functionals used in practical DFT (approximate DFT), unless special forms are taken into account. This is sometimes referred as overestimation of the delocalisation by approximate DFT. Zhang and Yang [83] showed that SIE in case of delocalized states with non integer number of electrons, e.g. in HS point, is much bigger than in case of localized ones, where an integer number of electrons is present, e.g. in LS point. SIE will always artificially stabilize the energy of systems having fractional number of electrons compared to the corresponding ones with integer number of electrons. It is also worthwhile to stress that relative stability of the states with partial occupation relative to the ones with integer occupation (delocalized vs. localized) is of interest not only in study of JT systems as such, but also in the field of chemical reactivity or mixed valence compounds.

To solve this problem and to obtain $E_{\mathrm{JT}}$, a multideterminental DFT approach is applied. We need two types of DFT calculations: (1) a single-point calculation imposing the high symmetry on the nuclear geometry and the low symmetry on the electron density. This is achieved by introducing an adequate occupation scheme of the MOs. This gives the energy of a Slater determinant with an integer electron orbital occupancy. (2) A geometry optimization in the lower symmetry. $E_{\mathrm{JT}}$ is the difference in these two energies. To obtain the energies of the degenerate states at HS one needs to evaluate the energies of all possible single determinants with integer occupations in HS geometry. Thus, both steps will be repeated for all the possible combinations of electronic states in $G_{\mathrm{LS}}$. The energy of vertical (Franck-Condon) transition $E_{\mathrm{FC}}$, is easily obtained in promoting the unpaired electron from the ground state to the first excited state for the ground state geometry. Our computational recipe, for the case of $\mathrm{VCl}_{4}$ is schematically drawn in Fig. 2.

In order to discuss the JT distortion on the adiabatic potential surface we define a vector $\mathbf{R}_{\mathrm{JT}}$ as the vector given by the displacements of the atoms from the high symmetry point defined by the $\mathbf{R}_{\mathrm{HS}}$. The JT radius, $R_{\mathrm{JT}}$ is given by the length of the distortion vector between the high symmetry and the minimum energy configuration.

$$
\mathbf{R}_{\mathrm{JT}}=\mathbf{R}_{\mathrm{HS}}-\mathbf{R}_{\mathrm{LS}}=R_{\mathrm{JT}} \mathbf{u}
$$




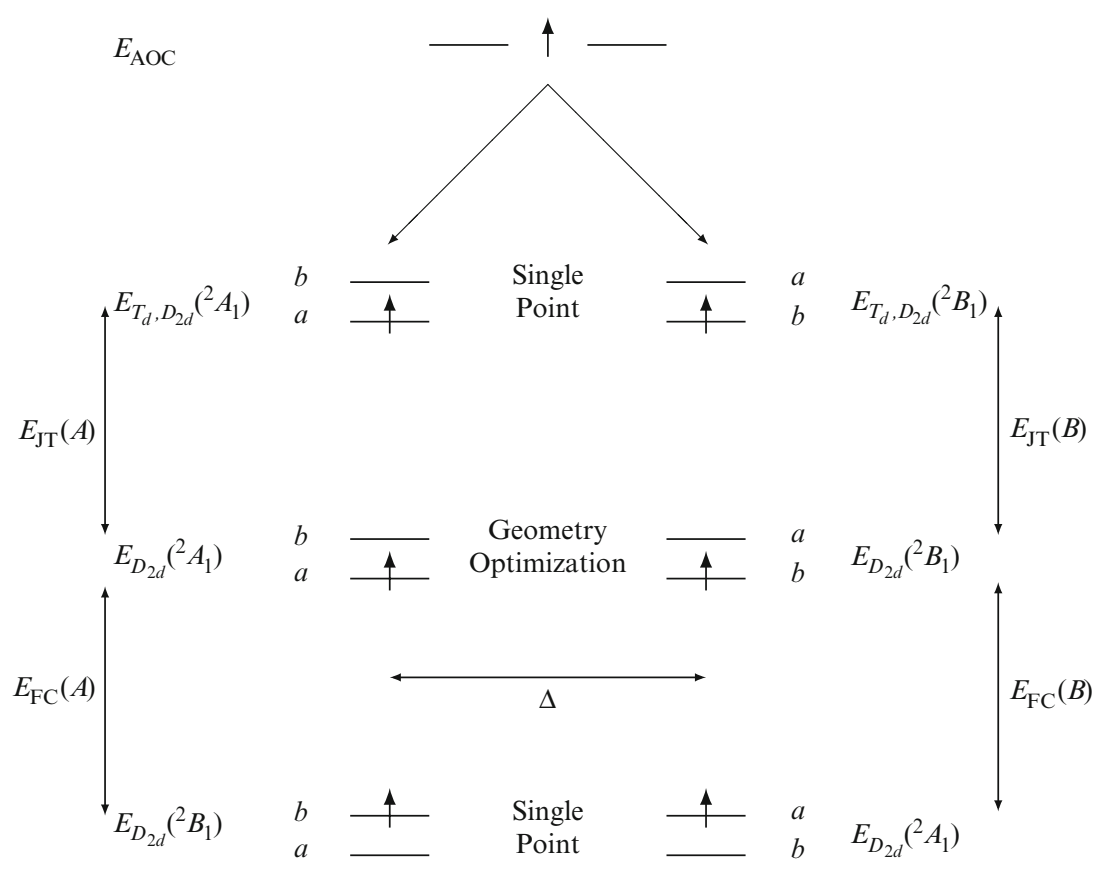

Fig. 2 Schematic representation of the calculation recipe in $T_{d}$ point group $-\mathrm{VCl}_{4}$

Let us summarize our calculation recipe:

1. AOC geometry optimization with fractional orbital occupation. This yields the HS geometry $\mathbf{R}_{\mathrm{HS}}$

2. Geometry optimization with the different LS electron distributions. This yields the different LS geometries $\mathbf{R}_{\mathrm{LS} \text {, min }}$ and $\mathbf{R}_{\mathrm{LS} \text {,TS }}$, and the different energies $E_{\mathrm{LS} \text {,min }}$ and $E_{\mathrm{LS}, \mathrm{TS}}$ that correspond to the minimum and to the transition state on the potential energy surface respectively

3. Single point calculation with fixed nuclear geometry $\mathbf{R}_{\mathrm{HS}}$ and different LS electron distributions with an integer SD occupations, resulting the energies $E_{\mathrm{HS}, \mathrm{LS} \text {,min }}$ and $E_{\mathrm{HS}, \mathrm{LS}, \mathrm{TS}}$. Energies for the different distributions should be equal

4. Single point calculation of the excited states with $\mathbf{R}_{\mathrm{LS}}$ to obtain $E_{\mathrm{FC}}$.

Combination of the calculated energies yield the JT parameters, $E_{\mathrm{JT}}, \Delta, E_{\mathrm{FC}}$.

$$
\begin{aligned}
& E_{\mathrm{JT}, \min }=E_{\mathrm{HS}, \mathrm{LS}, \min }-E_{\mathrm{LS}, \min }, \\
& E_{\mathrm{JT}, \mathrm{TS}}=E_{\mathrm{HS}, \mathrm{LS}, \mathrm{TS}}-E_{\mathrm{LS}, \mathrm{TS}}, \\
& \Delta=E_{\mathrm{LS}, \min }-E_{\mathrm{LS}, \mathrm{TS}}=E_{\mathrm{JT}, \min }-E_{\mathrm{JT}, \mathrm{TS}} .
\end{aligned}
$$

Within a harmonic approximation the JT distortion is given as a linear combination of displacements along all, $N_{a_{1}}$, totally symmetric normal coordinates in the 
LS conformation. The linear coefficients, or the weighting factors, $w_{\mathrm{HSk}}$, define the contribution of each of these normal modes, $\mathbf{Q}_{\mathrm{k}}$, to the distortion.

$$
\mathbf{R}_{\mathrm{JT}}=\sum_{\mathrm{k}=1}^{N_{a_{1}}} w_{\mathrm{HSk}} \mathbf{Q}_{\mathrm{k}} .
$$

Each of the totally symmetric normal modes contributes the energy $E_{\mathrm{k}}$ to the JT stabilisation, and $E_{\mathrm{JT}}$ can be expressed as the sum of these energy contributions, (23). Force at HS point, which drives the nuclei along $\mathbf{Q}_{\mathrm{k}}$ to the minimum is given by $\mathbf{F}_{\text {HSk }}(24) .{ }^{4}$

$$
\begin{gathered}
E_{\mathrm{JT}}=\sum_{\mathrm{k}=1}^{N_{a_{1}}} E_{\mathrm{k}}=\frac{1}{2} \sum_{\mathrm{k}=1}^{N_{a_{1}}} w_{\mathrm{HSk}}^{2} \mathbf{Q}_{\mathrm{k}}^{2} \lambda_{\mathrm{k}}, \\
\mathbf{F}_{\mathrm{HSk}}=w_{\mathrm{HSk}} \lambda_{\mathrm{k}} \mathbb{M}^{1 / 2} \mathbf{Q}_{\mathrm{k}} .
\end{gathered}
$$

Detailed discussion of this analysis is given in the Sect. 5 .

\section{Applications}

In this section we present the applications of DFT to discuss JT distortions. The results demonstrate that the computational recipe, described previously, Sect. 3, allows the calculation of the JT parameters, which are in good agreement with the experimental results. In this section, results of the analysis of the multimode JT effect are presented too.

\subsection{Tetrachlorovanadium(IV), $\mathrm{VCl}_{4}$}

Among the simplest of the $\mathrm{JT}$ molecules is $\mathrm{VCl}_{4}$, a tetrahedral molecule with a $\mathrm{d}^{1}$ configuration. It is characterized by a small JT effect. The method of calculation of the JT parameters using DFT was first developed on this system in our group [21] and it shows some important features.

In $T_{d}$ point group, a single electron occupies $e$ orbital. The electronic ground state is ${ }^{2} E$. After the symmetry descent to $D_{2 d}$ the later splits into ${ }^{2} A_{1}$ and ${ }^{2} B_{1}$. In order to obtain JT parameters calculation recipe discussed in Sect. 3 is applied. Calculation method is summarized in Fig. 2 and results are given in Table 2.

\footnotetext{
${ }^{4}$ In (23) and (24) eigenvectors $\mathbf{Q}_{\mathrm{k}}$, of the Hessian obtained in the LS minimum are expressed in generalized (mass-weighted) displacement coordinates, with eigenvalues $\lambda_{\mathrm{k}} ; \mathbb{M}$ is a diagonal $3 N \times 3 N$ matrix with atomic masses in triplicates as elements $\left(m_{1}, m_{1}, m_{1}, m_{2}, \ldots, m_{N}\right)$.
} 
Table 2 Results of the DFT calculations performed to analyse the JT effect of $\mathrm{VCl}_{4}$; energies (LDA) are given in $\mathrm{eV}$; the JT parameters $E_{\mathrm{JT}}$ and $\Delta$ are given in $\mathrm{cm}^{-1}$ and $R_{\mathrm{JT}}$ in $(\mathrm{amu})^{1 / 2} \AA$

\begin{tabular}{lccc}
\hline Occupation & State & Geometry & Energy \\
\hline$e^{0.5} e^{0.5}$ & ${ }^{2} E$ & $T_{d}$ & -21.7470 \\
$b_{1}^{0} a_{1}^{1}$ & ${ }^{2} A_{1}$ & $T_{d}$ & -21.6074 \\
$a_{1}^{0} b_{1}^{1}$ & ${ }^{2} B_{1}$ & $T_{d}$ & -21.6084 \\
$b_{1}^{0} a_{1}^{1}$ & ${ }^{2} A_{1}$ & $D_{2 d}$ & -21.6137 \\
$a_{1}^{0} b_{1}^{1}$ & ${ }^{2} B_{1}$ & $D_{2 d}$ & -22.6134 \\
$E_{J T}$ & ${ }^{2} A_{1}$ & & 50.8 \\
$E_{J T}$ & ${ }^{2} B_{1}$ & & 40.3 \\
$\Delta$ & & & 10.5 \\
$R_{\mathrm{JT}}$ & ${ }^{2} A_{1}$ & & 0.10 \\
$R_{\mathrm{JT}}$ & ${ }^{2} B_{1}$ & & 0.10 \\
\hline
\end{tabular}

As a starting point the geometry of $\mathrm{VCl}_{4}$ has been optimized in $T_{d}$ symmetry using an AOC calculation. This means that both $e$ orbitals carry 0.5 electron leading to a totally symmetric electron distribution. The second step is to carry out a calculation with fixed $T_{d}$ geometry occupying selectively one of the two degenerate orbitals. Finally a geometry optimization in $D_{2 d}$ symmetry is performed, corresponding to both ${ }^{2} A_{1}$ and ${ }^{2} B_{1}$ electronic ground states, leading to two different geometries and energies. Only due to the imposal of different electron distribution in two $D_{2 d}$ cases, the calculations give rise to the simulation of the JT distortion. The results of this calculation are shown in Table 2.

We notice that the energies $E\left({ }^{2} A_{1}, T_{d}\right)$ and $E\left({ }^{2} B_{1}, T_{d}\right)$ are not equal. This inequality is due to the nature of the numerical integration grid involved in DFT calculations [28]. This is generally observed also if symmetry arguments impose equal energy. In this cases energy difference between two is negligible, e.g. in the case of cobaltocene (see Sect. 4.3). From the Table 2 it is evident why the two calculations with $T_{d}$ nuclear geometries and $D_{2 d}$ electron densities have been performed. Comparing the final energies in LS with the one obtained from AOC calculation in $T_{d}$ would give a misleading result, that there is no JT effect. The electron distribution in the nondistorted $\mathrm{VCl}_{4}$ is different from that in distorted one. The electron interaction term in the total energy is also different. In order to compare the two LS geometries with one in the HS, the unpaired electron needs to be distinguishably placed in one of the two $e$ orbitals, as done in our calculation scheme.

Within $3 N-6=9$ normal modes only one pair of $e$ and one $a_{1}$ modes have non zero linear vibronic coupling constant. Thus this can be the simplest case of the multimode problem, with possibly two JT active vibrations. Applying our method for the analysis of the different contributions of the normal coordinates, Sect. 5, we find that the contribution of the $e$ mode to the distortion is more than $99 \%$, which is in agreement with usual consideration of $\mathrm{VCl}_{4}$ system as an ideal, single mode problem. This also justifies full potential energy surface calculation along the JT active component of the degenerate vibration, $\mathbf{Q}_{a}$ for both electronic states. In the 
case of simple molecules, e.g. tetrahedral $\mathrm{VCl}_{4}$, it is possible to have analytical expression for the displacements, which can be found in e.g. [46].

This vibration is illustrated in Fig. 3, potential energy surface calculation along it on Fig. 4, and the Mexican-hat-like plot on Fig. 5.

In summary this shows that DFT calculations for this simple molecule, with a relatively small JT effect, yields results in good agreement with the experiments $[4,16,48,61] . E_{\mathrm{JT}}=50.8 \mathrm{~cm}^{-1}$ is obtained by the DFT calculation and the

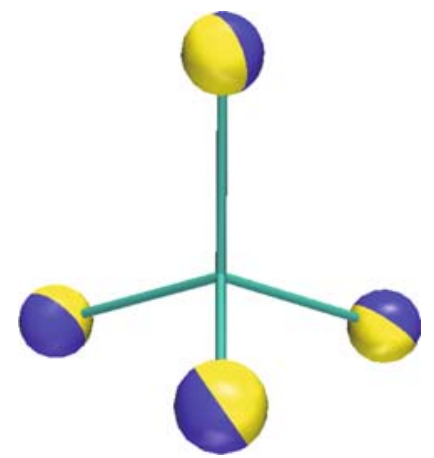

Fig. 3 Vibrational energy distribution representation of the JT active vibration of $\mathrm{VCl}_{4}$. The different colours indicate the direction of the displacement vector; the volume of the spheres is proportional to the contribution made by the individual nuclei to the energy of the vibrational mode

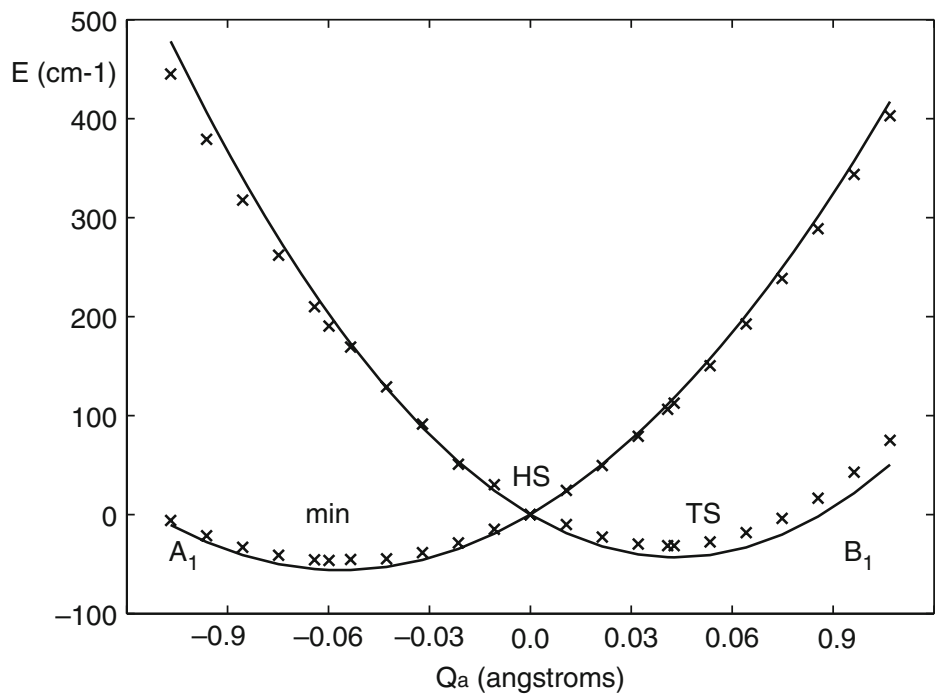

Fig. $4 \mathrm{VCl}_{4}$ potential energy surface calculation along the JT active vibration $\mathbf{Q}_{a}(\AA)$ (times) and least square fitting of the data (minus); energies are given in $\mathrm{cm}^{-1}$ relative to the HS point 


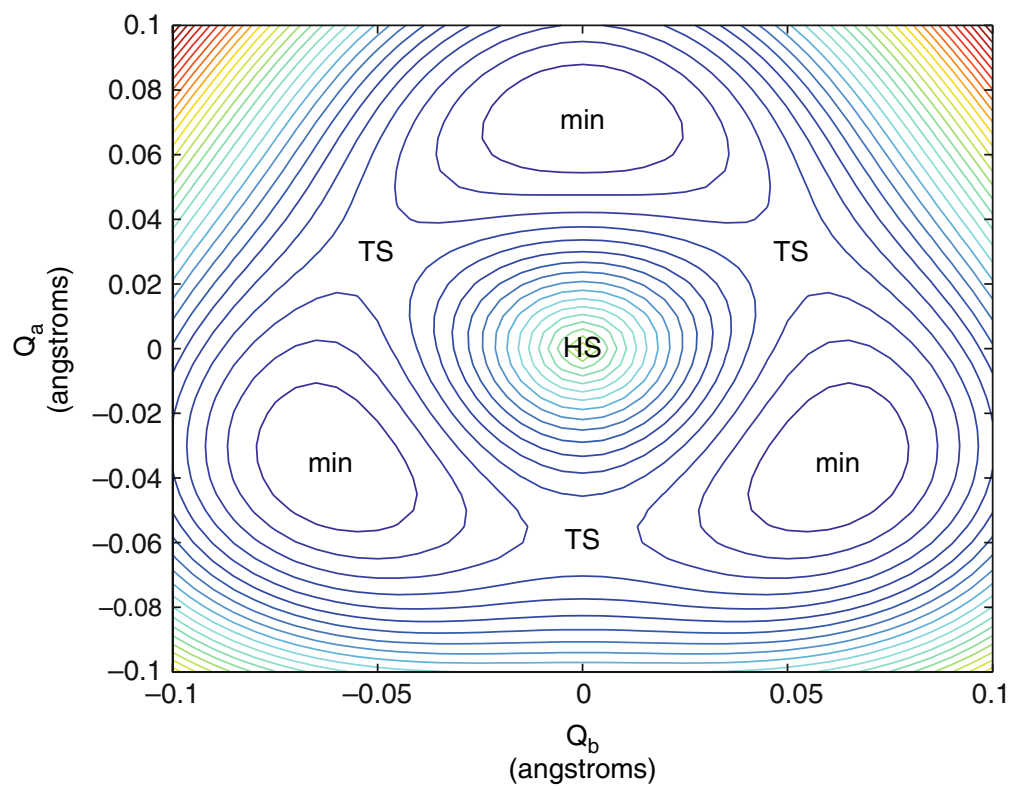

Fig. $5 \mathrm{VCl}_{4}$ Mexican-hat-like contour plot of the adiabatic potential energy in the space of $\mathbf{Q}_{a}$ and $\mathbf{Q}_{b}$ components of the JT active $e$ vibration

experimental value lies between 30 and $80 \mathrm{~m}^{-1}[4,16,48,61]$. These results confirm the dynamic character of the JT effect.

\subsection{Cyclopentadienyl Radical}

Cyclopentadienyl radical is one of the most studied JT active molecules, both experimentally and theoretically. Theoretical works trace back to Andy Liehr in 1956 [54]. They span many different methods $[5,12,17,26,43,45,50,54,59,72,85]$ during the years. The values of $E_{\mathrm{JT}}$ obtained are summarised in Table 3. The JT effect was discussed using various models, (1) the classical perturbation model as in the work of Liehr [54] (2) models based on the analysis of spectra as in the works of Miller et al. [5, 6] or Stanton et al. [45], (3) Valence Bond (VB) model [85], or (4) vibronic coupling density analysis [67]. Somehow surprisingly there was to our knowledge no attempt to use DFT to analyse the JT effect in this system up to now. As it can be seen from the Table 3 our multideterminental DFT approach gives the value of $1,253 \mathrm{~cm}^{-1}$ for $E_{\mathrm{JT}}$ which is in excellent agreement with the experimental one of $1,237 \mathrm{~cm}^{-1}$ [6]. The various other theoretical methods give different results ranging from 495 to $5,072 \mathrm{~cm}^{-1}$. Studies of Miller et al. $[5,6]$ who used complete active space methods $\left(E_{\mathrm{JT}}=2147 \mathrm{~cm}^{-1}\right)$ and dispersed fluorescence spectroscopy $\left(E_{\mathrm{JT}}=1237 \mathrm{~cm}^{-1}\right)$, as well as fitting of ab initio calculations to the spectra 
Table 3 Summary of various computational methods used to study the JT effect in $\mathrm{C}_{5} \mathrm{H}_{5}$; $E_{\mathrm{JT}}$ is given in $\mathrm{cm}^{-1}$

\begin{tabular}{ll}
\hline Method $^{a} /$ Basis set & $E_{\mathrm{JT}}$ \\
\hline Semiempirical-MO [54] & 560 \\
Semiempirical-MO [72] & 728 \\
Semiempirical-MO [43] & 495 \\
HF/STO-3G [59] & 5,072 \\
CI/STO-3G [17] & 2,484 \\
HF/6-311+G* [26] & 1,452 \\
MP2/6-311+G* [26] & 3,065 \\
MP4/6-311+G* [26] & 2,581 \\
CCSD/6-311+G* [26] & 1,613 \\
CCSD(T)/6-311+G* [26] & 1,613 \\
CASSCF/cc-PVDZ [12] & 2,139 \\
CASSCF/6-31G* [5] & $2,147 / 1,463$ \\
CASSCF/cc-PVDZ [50] & 1,665 \\
CISD/cc-PVDZ [85] & 2,553 \\
EOMIP-CCSD/DZP [45] & 1,581 \\
DFT(LDA)/TZP & 1,253 \\
DFT(PW91)/TZP & 1,326 \\
Exp. [6] & 1237
\end{tabular}

${ }^{a}$ Acronyms used for the calculation methods: $H F$ Hartree-Fock; $C I$ Configuration Interaction; $M P N$ Møller-Plesset Perturbation Theory of order N for electron correlation; $\operatorname{CSD}(T)$ Coupled Cluster Single, Double (Triple) excitations; CASSCF Complete-Active-Space SCF; CISD Single and Double excitations, single reference CI method; EOMIP-CCSD Equation-of-motion ionization potential coupled-cluster single, double excitations; LDA Local Density Approximation; PW91 Generalized Gradient Approximation in the form given by Perdew-Wang

${ }^{b}$ Multideterminental DFT - this work

$\left(E_{\mathrm{JT}}=1,463 \mathrm{~cm}^{-1}\right)$ are considered to be benchmark results for the determination of the JT parameters. They also identified three dominant normal modes necessary to explain their results. These were recently confirmed by Stanton et al. using Equation-of-motion ionization potential coupled-cluster (EOMIP-CCSD) calculations [45]. Thus, this system is a good test case for both our multideterminental DFT approach in studies of the JT effect and for our model of the analysis of the multimode JT effect.

The ground electronic state of $\mathrm{C}_{5} \mathrm{H}_{5}$ in $D_{5 h}$ symmetry is ${ }^{2} E_{1}^{\prime \prime}$, with three electrons occupying the doubly degenerate orbital (one hole). Using group theory it is easy to show, see Sect. 2 that the distortion coordinate is $e_{2}^{\prime}$. The descent in symmetry goes to $C_{2 v}$. The electronic state ${ }^{2} E_{1}^{\prime \prime}$ splits into ${ }^{2} A_{2}$ and ${ }^{2} B_{1}$ and the JT active distortion $e_{2}^{\prime}$ splits into $a_{1}$ and $b_{2}$. The results of the DFT calculation are summarised in the Table 4.

The difference in the JT energies for the different electronic states is only $3.2 \mathrm{~cm}^{-1}$. This confirms results of the analysis by group theory, see Sect. 2, that 
Table 4 Results of the DFT calculations performed to analyse the JT effect of $\mathrm{C}_{5} \mathrm{H}_{5}$; energies (LDA) are given in $\mathrm{eV}$; the JT parameters $E_{\mathrm{JT}}$ and $\Delta$ are given in $\mathrm{cm}^{-1}$ and $R_{\mathrm{JT}}$ in $(\mathrm{amu})^{1 / 2} \AA$

\begin{tabular}{lcrr}
\hline Occupation & State & Geometry & Energy \\
\hline$e^{0.75} e^{0.75}$ & ${ }^{2} E$ & $D_{5 h}$ & -64.6740 \\
$b_{1}^{2} a_{1}^{1}$ & ${ }^{2} A_{1}$ & $D_{5 h}$ & -64.6529 \\
$a_{1}^{2} b_{1}^{1}$ & ${ }^{2} B_{1}$ & $D_{5 h}$ & -64.6523 \\
$b_{1}^{2} a_{1}^{1}$ & ${ }^{2} A_{1}$ & $C_{2 v}$ & -64.8079 \\
$a_{1}^{2} b_{1}^{1}$ & ${ }^{2} B_{1}$ & $C_{2 v}$ & -64.8077 \\
$E_{J T}$ & ${ }^{2} A_{1}$ & & $1,250.2$ \\
$E_{J T}$ & ${ }^{2} B_{1}$ & & $1,253.4$ \\
$\Delta$ & & & 3.2 \\
$R_{J T}$ & ${ }^{2} A_{1}$ & & 0.17 \\
$R_{J T}$ & ${ }^{2} B_{1}$ & & 0.18 \\
\hline
\end{tabular}

Table 5 Analysis of the JT multimode problem in $\mathrm{C}_{5} \mathrm{H}_{5}$ radical by LS totally symmetric normal modes in harmonic approximation. Frequencies of normal modes are in $\mathrm{cm}^{-1}$ as obtained from ADF $[1,40,76]$ calculations; contribution of the normal mode $\mathbf{Q}_{\mathrm{k}}$ to the $\mathbf{R}_{\mathrm{JT}}$ is given by $w_{\mathrm{k}}$ (linear coefficients in (22)); $c_{\mathrm{k}}$ linear coefficients $\left(w_{\mathrm{k}}\right)$ normalized to $1 ; E_{\mathrm{k}}$ energy contribution of $\mathbf{Q}_{\mathrm{k}}$ to the $E_{\mathrm{JT}}$ calculated in harmonic approximation, (23) in $\mathrm{cm}^{-1} ; F_{\mathrm{k}}$ force along $\mathbf{Q}_{\mathrm{k}}$ at HS point, calculated in harmonic approximation, $(24)$ in $10^{3} \mathrm{~N}$; experimental value $E_{\mathrm{k}}^{\text {exp }}$, and two theoretical values $E_{\mathrm{k}}^{t 1}$ and $E_{\mathrm{k}}^{t 2}$ in $\mathrm{cm}^{-1}$ from $[5,6] . E_{\mathrm{JT}}(\mathrm{DFT})$, in $\mathrm{cm}^{-1}$, from multideterminental DFT, this work

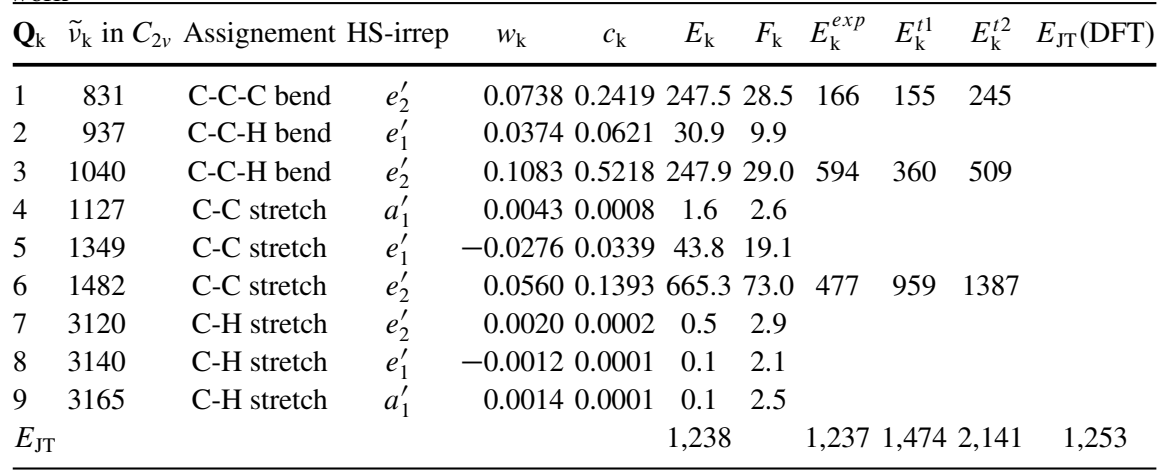

the quadratic coupling constant, $G_{\mathrm{ij}}^{\mathrm{kl}}$ is zero, and there is no warping of the Mexican hat. In order to analyse multimode character of the JT distortion in $\mathrm{C}_{5} \mathrm{H}_{5}$ we express the distortion as a linear combination of all totally symmetric normal modes in the LS energy minimum. Details of the procedure will be given later in Sect. 5, and only the results are presented in Table 5.

We are able to identify the three most important vibrations contributing to the JT distortion, vibrations 1,3 and 6 , in agreement with previous studies [5,6]. Comparing our results to the experimental one we may note that vibrations 3 and 6 are contributing approximately the same amount to the $E_{\mathrm{JT}}$. While the other authors considered only those three normal modes, in our model all vibrations that can contribute to the JT distortion are included. Our model is completely theoretical without 


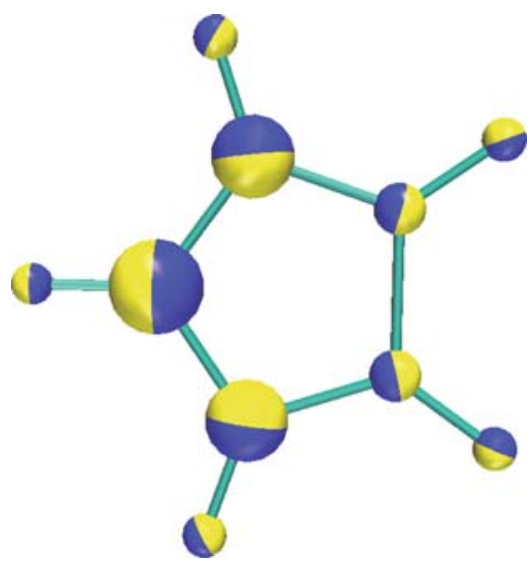

Fig. 6a vibration 1 (C-C-C bend)

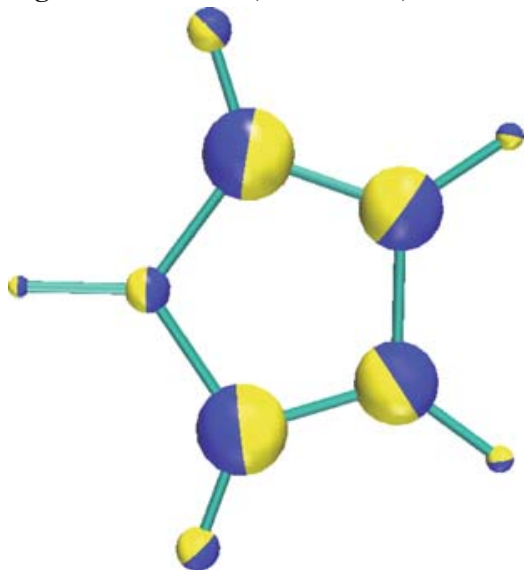

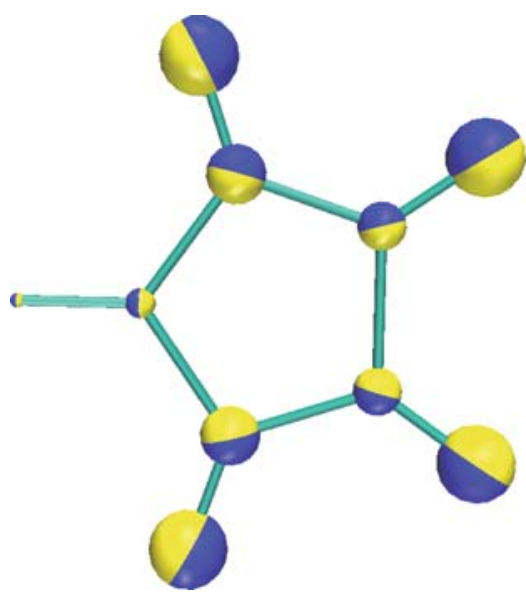

Fig. 6 b vibration 3 (C-C-H bend)

Fig. 6c vibration 6 (C-C stretch)

Fig. 6 Vibrational energy distribution representation of the three most important $a_{1}$ vibrations in $C_{2 v}$ symmetry of $\mathrm{C}_{5} \mathrm{H}_{5}$, corresponding to the three $e_{2}^{\prime} \mathrm{JT}$ active vibrations in $D_{5 h}$ symmetry. The different colours indicate the direction of the displacement vector; the volume of the spheres is proportional to the contribution made by the individual nuclei to the energy of the vibrational mode

any fitting to the experimental data. Three most dominant vibrations are presented in the Fig. 6.

Vibrations 1, 3, 6 contribute $90 \%$ to the JT distortion. Vibrations 2 and 5, which correspond to the $e_{1}^{\prime}$ irreps in $D_{5 h}$ around $10 \%$. They are JT active in the second order, and accordingly not negligible. This is because the vibrations are all of the same type, in plane ring deformation, as the ones corresponding to the $e_{2}^{\prime}, \mathrm{C}-\mathrm{C}-\mathrm{C}$ bend and $\mathrm{C}-\mathrm{C}$ stretch, thus influencing the $\mathrm{C}-\mathrm{C}$ bonding in a similar way. 


\subsection{Cobaltocene}

The high symmetry conformation of metallocenes can be either $D_{5 h}$ if the two rings are eclipsed or $D_{5 d}$ if the two rings are staggered. In both cases the symmetry arguments are the same as for an intermediate structure of $D_{5}$ symmetry. The ground electronic state of cobaltocene in $D_{5 h}$ symmetry is ${ }^{2} E_{1}^{\prime \prime},{ }^{2} E_{1 g}$ in $D_{5 d}$, with a single electron in the doubly degenerate orbital. Using group theory it is easy to show, see Sect. 2 that the distortion coordinates are of $e_{2}^{\prime}$ irreps in the eclipsed and of $e_{2 g}$ in the staggered conformation, and the descent in symmetry goes to $C_{2 v}$ and $C_{2 h}$ respectively. The electronic states will split in $D_{5 h} E_{1}^{\prime \prime}$ into $A_{2}$ and $B_{1}$, in $D_{5 d} E_{1 g}$ into $A_{g}$ and $B_{g}$. Respectively, the JT active distortion $e_{2}^{\prime}$ splits into $a_{1}$ and $b_{2}$ and $e_{2 g}$ into $a_{g}$ and $b_{g}$.

This system is more complicated than the previous ones, because an internal rotation of the rings is present. Our study [86] showed that this rotation does not influence the JT distortion. In the low symmetry, $C_{2 v}$ for the eclipsed, $C_{2 h}$ for the staggered conformation, the structure of the rings is nearly identical. This strongly suggests that the energy barrier for the rotation of the rings is small compared to the $E_{\mathrm{JT}}$. This was verified calculating the energy profile for the ring rotation. The energy barrier for the internal rotation of the rings, from eclipsed to staggered conformation, is estimated to be around $240 \mathrm{~cm}^{-1}$ in both high and low symmetries, similar to the energy for the rotation of the rings in ferrocene [13]. Cobaltocene in $D_{5 h}$ symmetry is approximately $160 \mathrm{~cm}^{-1}$ more stable than in $D_{5 d}$. This is in agreement with results of previous DFT calculations on metallocenes [75, 82]. The energy difference between the low symmetry conformations $C_{2 v}$ and $C_{2 h}$ obtained by descent in symmetry from $D_{5 h}$ and $D_{5 d}$ is similar. This is summarised in the Fig. 7.

Cobaltocene has been subject of wide research $[3,4,22,34,52,58,74,80]$, but only recently a detailed analysis [86] of the JT distortion has been carried out. Calculations were done for both the eclipsed and the staggered conformations, giving similar results. In this paper we will present only the results for the more stable of the two, i.e. for the eclipsed case. Details for both eclipsed and staggered conformation can be found in [86]. As already indicated in previous Sect. 3, DFT produces a totally symmetric electron distribution if each $e_{1}^{\prime \prime}$ orbital carries 0.5 electrons. There are two distinct ways to accommodate the single electron in $C_{2 v}$ symmetry, i.e. $a_{2}^{1} b_{1}^{0}$ ( ${ }^{2} A_{2}$ electronic state) or $b_{1}^{1} a_{2}^{0}\left({ }^{2} B_{1}\right.$ electronic state). One of the states is stabilized by a distortion along $+\mathbf{R}_{\mathrm{JT}}$, the other along $-\mathbf{R}_{\mathrm{JT}}$. Thus, DFT calculations corresponding to both of these occupations, as well as to the $G_{\mathrm{HS}}=D_{5 h}$ and $G_{\mathrm{LS}}=C_{2 v}$ geometries, are carried out, leading to the values of JT stabilization energies, $E_{\mathrm{JT}}(A)$ and $E_{\mathrm{JT}}(B)$. Results are tabulated in Table 6.

The JT stabilization energy is $814.2 \mathrm{~cm}^{-1}$ in good agreement with the value of $1050 \mathrm{~cm}^{-1}$ estimated from the solid state EPR [4,22]. The experimental results strongly depend on the diamagnetic host matrix, thus making experimental determination of the JT parameters difficult. The JT energies for the different electronic states are almost exactly the same, the difference is only $0.7 \mathrm{~cm}^{-1}$, smaller 


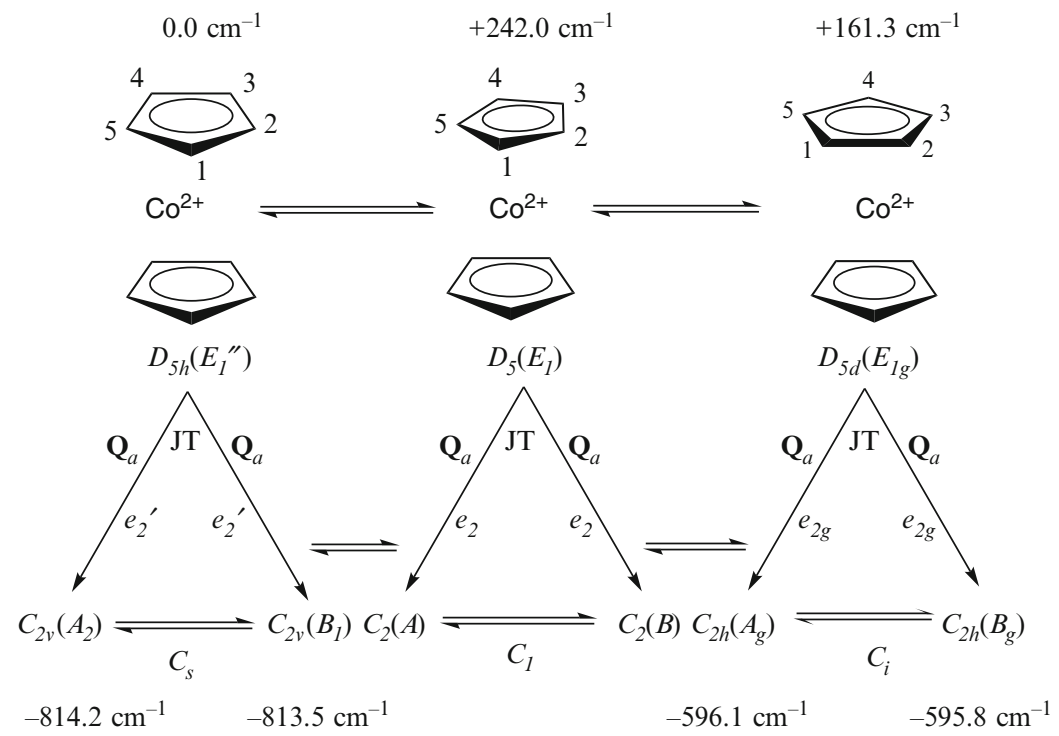

Fig. 7 Summary of the JT effect in cobaltocene. Symmetries of the corresponding geometries, electronic states and normal coordinates, numbering of $\mathrm{C}$ atoms in the cyclopentadienyl rings, as well as the relative energies of the different structures is given

Table 6 Results of the DFT calculations performed to analyse the JT effect of cobaltocene; energies (LDA) are given in $\mathrm{eV}$; the JT parameters $E_{\mathrm{JT}}$ and $\Delta$ are given in $\mathrm{cm}^{-1}$ and $R_{\mathrm{JT}}$ in $(\mathrm{amu})^{1 / 2} \AA$

\begin{tabular}{lccc}
\hline Occupation & State & Geometry & Energy \\
\hline$e^{0.5} e^{0.5}$ & ${ }^{2} E$ & $D_{5 h}$ & -142.28971 \\
$a_{2}^{0} b_{1}^{0}$ & ${ }^{2} A_{2}$ & $D_{5 h}$ & -142.26105 \\
$b_{1}^{1} a_{2}^{0}$ & ${ }^{2} B_{1}$ & $D_{5 h}$ & -142.26113 \\
$a_{2}^{1} b_{1}^{0}$ & ${ }^{2} A_{2}$ & $C_{2 v}$ & -142.36200 \\
$b_{1}^{1} a_{2}^{0}$ & ${ }^{2} B_{1}$ & $C_{2 v}$ & -142.36199 \\
$E_{J T}$ & ${ }^{2} A_{2}$ & & 814.2 \\
$E_{J T}$ & ${ }^{2} B_{1}$ & & 813.5 \\
$\Delta$ & & & 0.7 \\
$R_{J T}$ & ${ }^{2} A_{2}$ & & 0.35 \\
$R_{J T}$ & ${ }^{2} B_{1}$ & & 0.35 \\
\hline
\end{tabular}

then the precision of the calculations. As expected, based on group theoretical considerations, Sect. 2, there is no warping of the potential energy surface.

Cobaltocene is an another example of the multimode JT system. There are six pairs of $e_{2}^{\prime}$ vibrations which are first order JT active, four $a_{1}^{\prime}$ and six pairs of $e_{1}^{\prime}$ vibrations which are second order JT active. They become all totally symmetric in $C_{2 v}$ symmetry (one component of each pair in the case of the degenerate vibrations). Thus in $C_{2 v}$ symmetry we have 16 totally symmetric vibrations. As already pointed 
Table 7 Analysis of the JT multimode problem in cobaltocene by LS totally symmetric normal modes in harmonic approximation. Frequencies of normal modes are in $\mathrm{cm}^{-1}$ as obtained from ADF $[1,40,76]$ calculations; contribution of the normal mode $\mathbf{Q}_{\mathrm{k}}$ to the $\mathbf{R}_{\mathrm{JT}}$ is given by $w_{\mathrm{k}}$ (linear coefficients in (22)); $c_{\mathrm{k}}$ linear coefficients $\left(w_{\mathrm{k}}\right)$ normalized to $1 ; E_{\mathrm{k}}$ energy contribution of $\mathbf{Q}_{\mathrm{k}}$ to the $E_{\mathrm{JT}}$ calculated in harmonic approximation, (23) in $\mathrm{cm}^{-1} ; F_{\mathrm{k}}$ force along $\mathbf{Q}_{\mathrm{k}}$ at HS point, calculated in harmonic approximation, (24) in $10^{3} \mathrm{~N} ; E_{\mathrm{JT}}(\mathrm{DFT})$, in $\mathrm{cm}^{-1}$, from multideterminental DFT [86]

\begin{tabular}{|c|c|c|c|c|c|c|c|c|}
\hline $\mathbf{Q}_{\mathrm{k}}$ & $\tilde{v}_{\mathrm{k}}$ in $C_{2 v}$ & Assignment & HS-irrep & $w_{\mathrm{k}}$ & $c_{\mathrm{k}}$ & $E_{\mathrm{k}}$ & $F_{\mathrm{k}}$ & $E_{\mathrm{JT}}(\mathrm{DFT})$ \\
\hline 1 & 153 & skeletal bending & $e_{1}^{\prime}$ & 0.0035 & 0.0003 & 0.02 & 0.1 & \\
\hline 2 & 292 & ring-metal stretch & $a_{1}^{\prime}$ & -0.0172 & 0.0080 & 1.95 & 1.6 & \\
\hline 3 & 405 & ring tilt & $e_{1}^{\prime}$ & 0.0097 & 0.0025 & 0.03 & 4.2 & \\
\hline 4 & 587 & out-of-plane ring deformation & $e_{2}^{\prime}$ & -0.1550 & 0.6495 & 475.49 & 48.2 & \\
\hline 5 & 762 & $\mathrm{C}-\mathrm{H}$ wagging & $a_{1}^{\prime}$ & -0.0147 & 0.0058 & 2.11 & 2.0 & \\
\hline 6 & 825 & C-H wagging & $e_{1}^{\prime}$ & -0.0181 & 0.0082 & 4.12 & 3.8 & \\
\hline 7 & 830 & in-plane ring distortion & $e_{2}^{\prime}$ & -0.0621 & 0.1044 & 118.86 & 32.4 & \\
\hline 8 & 869 & $\mathrm{C}-\mathrm{H}$ wagging & $e_{2}^{\prime}$ & -0.0657 & 0.1166 & 66.22 & 17.4 & \\
\hline 9 & 976 & $\mathrm{C}-\mathrm{H}$ bending & $e_{1}^{\prime}$ & 0.0084 & 0.0019 & 1.44 & 3.1 & \\
\hline 10 & 1031 & in-plane $\mathrm{C}-\mathrm{H}$ bending & $e_{2}^{\prime}$ & 0.0547 & 0.0809 & 55.77 & 15.5 & \\
\hline 11 & 1126 & $\begin{array}{l}\text { ring breathing mode } \\
\text { (C-C stretch) }\end{array}$ & $a_{1}^{\prime}$ & 0.0002 & 0.0000 & 0.00 & 0.3 & \\
\hline 12 & 1367 & C-C stretch & $e_{2}^{\prime}$ & 0.0209 & 0.0118 & 47.86 & 35.9 & \\
\hline 13 & 1397 & C-C stretch & $e_{1}^{\prime}$ & 0.0185 & 0.0093 & 34.15 & 30.0 & \\
\hline 14 & 3136 & C-H stretch & $e_{2}^{\prime}$ & -0.0017 & 0.0001 & 0.46 & 3.5 & \\
\hline 15 & 3148 & C-H stretch & $e_{1}^{\prime}$ & -0.0009 & 0.0000 & 0.13 & 1.9 & \\
\hline 16 & 3166 & C-H stretch & $a_{1}^{\prime}$ & -0.0002 & 0.0000 & 0.00 & 0.4 & \\
\hline$E_{\mathrm{JT}}$ & & & & & & 808.6 & & 814.2 \\
\hline
\end{tabular}

out in order to analyse JT distortion in terms of the contribution of different normal coordinates, we express the distortion as a linear combination of all totally symmetric normal modes in the low symmetry $\left(C_{2 v}\right)$ minimum energy conformation. The result is given in Table 7. Assignment of the vibrations is given according to the normal coordinate analysis of the ferrocene and ruthenocene $[2,13,20,68]$.

The main contribution to the JT distortion arises from the four $e_{2}^{\prime}$ type vibrations (labelled as 4, 7, 8 and 10 in Table 7). They contribute to about $95 \%$ of the total JT distortion vector. The four vibrations are: the out-of-plane ring distortion, 4 , the in-plane ring distortion, 7, the $\mathrm{C}-\mathrm{H}$ wagging (the out-of-plane $\mathrm{C}-\mathrm{H}$ bending), 8 , and in-plane $\mathrm{C}-\mathrm{H}$ bending, 10. These vibrations are illustrated in Fig. 8, using the vibrational energy distribution representation [44]. The analysis shows, that the contribution of low energy skeletal vibrations (1 to 3 ) and the high energy vibrations (C-H stretch 14 to 16 ) is almost negligible. The JT important $e_{2}^{\prime}$ vibrations, and hence the JT distortion, is predominantly located in the five-member rings. The main contribution is the out-of-plane deformation of cyclopentadienyl ring (vibration 4). This is expected because this normal coordinate minimizes antibonding interactions between the cyclopentadienyl ring orbitals and the single occupied metal $d$ orbital. The symmetry of the electronic ground state in HS point directs 


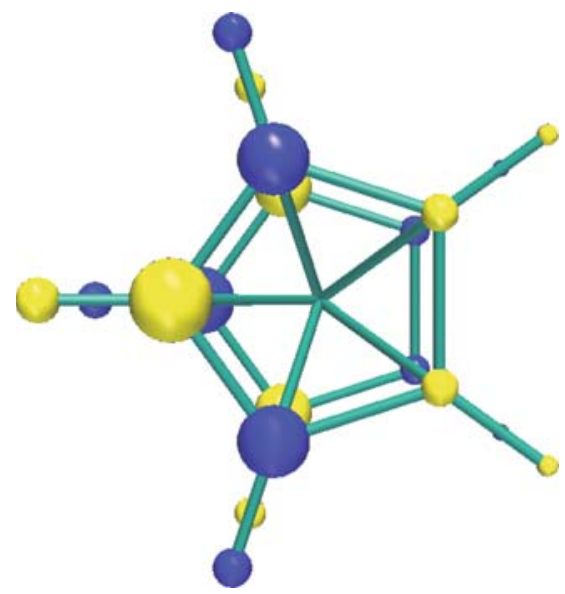

Fig. 8a vibration 4 (out-of-plane ring deformation)

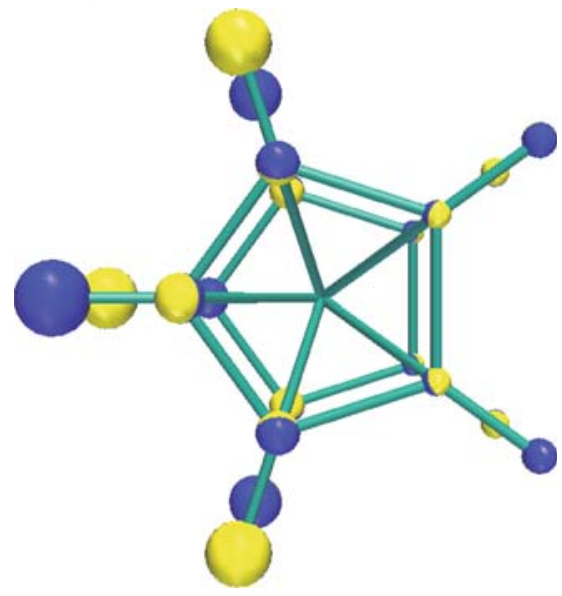

Fig. 8c vibration 8 (C-H wagging)

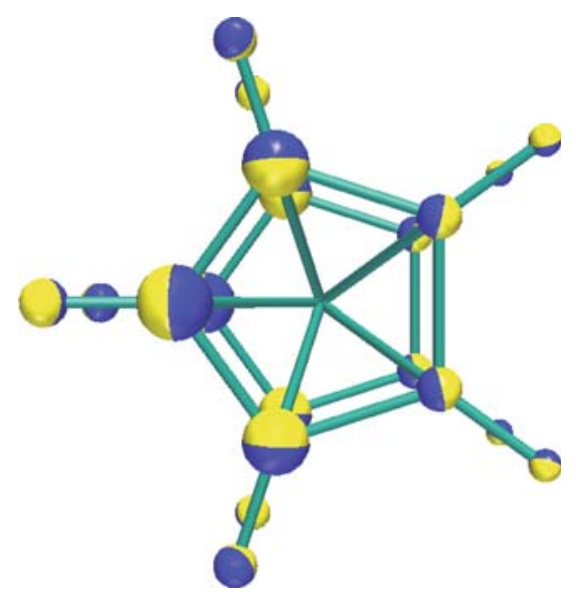

Fig. 8b vibration 7 (in-plane ring distortion)

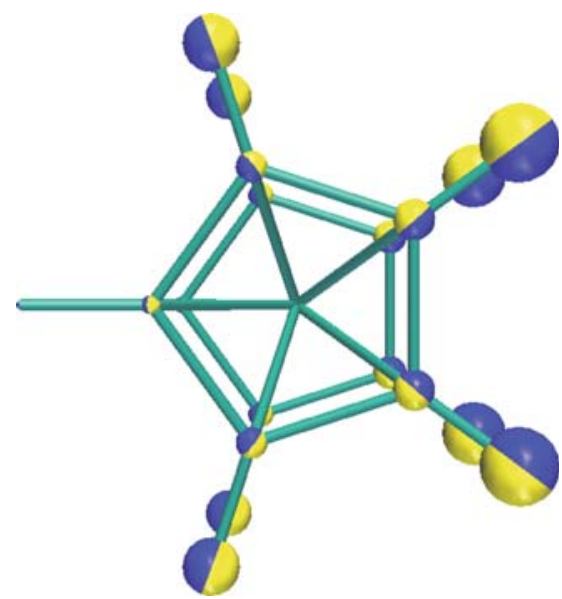

Fig. 8d vibration 10 (in-plane C-H bending)

Fig. 8 Vibrational energy distribution representation of the four most important $a_{1}$ vibrations in $C_{2 v}$ symmetry of cobaltocene, corresponding to the four $e_{2}^{\prime}$ vibrations in $D_{5 h}$ symmetry. The different colours indicate the direction of the displacement vector; the volume of the spheres is proportional to the contribution made by the individual nuclei to the energy of the vibrational mode

the distortion in a way of perturbing the aromaticity of the two rings. The multimode analysis gives a direct insight into microscopic origin of the distortion and into counterplay between the energy gain due to the JT effect and energy loss due to the out-of-plane distortion of the ligands.

$e_{1}^{\prime}$ vibrations, that are JT active in second order only are almost not contributing, except the $\mathrm{C}-\mathrm{C}$ stretch, vibration 13. The situation is different when comparing to 
the $\mathrm{C}_{5} \mathrm{H}_{5}, 4.2$. This is due to the fact that in cobaltocene $e_{2}^{\prime}$ and $e_{1}^{\prime}$ vibrations are of different type, $e_{2}^{\prime}$ are located in the ligands, while $e_{1}^{\prime}$ are mainly skeletal deformations. In $\mathrm{C}_{5} \mathrm{H}_{5}$ they are of the same kind, thus influencing bonding in a analogous way.

$E_{\mathrm{JT}}$ calculated from the LS normal coordinate analysis is in the excellent agreement with previously calculated one using multideterminental approach. In $\mathrm{C}_{5} \mathrm{H}_{5}$ both the JT electronic deformation and nuclear displacements are localized in the rings, while in $\mathrm{CoCp}_{2}$ the first is localized on the central metal ion and the latter on the $\mathrm{Cp}$ rings. In $\mathrm{C}_{5} \mathrm{H}_{5}$ contributions originating from the second order JT active vibrations, $e_{1}^{\prime}$, are consequently not negligible.

The mixing of the totally symmetric vibrations in lower symmetry is expected to increase with increasing deviation from the high symmetry geometry. It is interesting to see how the composition of the distortion vector changes along the minimal energy path. The later is defined as the steepest descent path [37,38], down from the JT, HS, cusp to the local energy minimum, LS. The former is easily calculated using the Intrinsic Reaction Coordinate (IRC) algorithm as implemented by Deng and Ziegler $[29,30]$ in the ADF program package $[1,40,76]$. There is a complete analogy between the JT distortion and chemical reaction paths, thus it is possible to use the same algorithms previously developed for the analysis of reaction paths. The JT distortion path is totally symmetric reaction path in the LS potential energy surface, connecting HS, JT cusp, and LS energy minimum. The high symmetry point has a nonzero gradient, thus, the first step is computed in direction of the steepest descent and not in the direction of a negative Hessian eigenvector as usually in IRC calculations starting from the transition states. The path is than computed by taking steps of adequate size and by optimizing all atomic coordinates orthogonal to it. During the calculation $C_{2 v}$ symmetry is conserved, and it is taken into account that one electronic state corresponds to the forward path, and the other to the backward path. IRC calculations for the eclipsed conformation of the rings are summarized in Fig. 9 together with the direct path. It can be seen that these two ways are not significantly different. Changes of the contributions of the four dominant vibrations along the IRC path are represented in Fig. 10.

The significance of different normal coordinates is not the same at the beginning step and at the minimum. Figure 10 shows that the composition of the distortion vector changes along the minimal energy path. In the beginning, the contribution of in-plane $\mathrm{C}-\mathrm{H}$ bending, 10, is also important, but as the distortion deviates from the high symmetry point its contribution decreases. The opposite is true for the lowest energy $e_{2}^{\prime}$ vibration 4 , the out-of-plane ring deformation, which is indeed the most important one. The contribution of $\mathrm{C}-\mathrm{H}$ wagging is also becoming more important. On the first sight, it might be surprising, that the softest of the four modes makes the largest contribution. This indicates that the distortion along the corresponding normal coordinate is larger than for any other one. One can see that the first point along the IRC path is already giving $2 / 3$ of the JT stabilisation. Thus, although IRC calculation gives the information that different contributions change along the reaction path, the information from the first, infinitesimally small, step is missing. This is due to the fact that IRC algorithm is implemented to locate the minima, reactants 


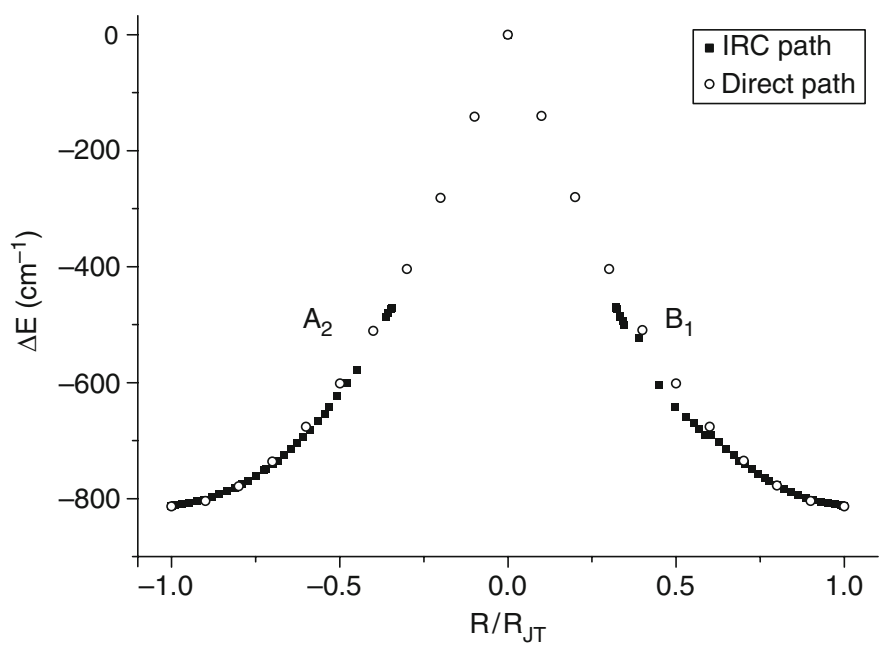

Fig. 9 IRC calculation (filled squares) and direct path (open circles) from the high symmetry cusp, in $C_{2 v}$ symmetry (eclipsed conformation of the rings); forward direction correspond to the ${ }^{2} B_{1}$ electronic state and backward direction to the ${ }^{2} A_{2}$ electronic state; energies are given in $\mathrm{cm}^{-1}$ relative to the $\mathrm{HS}$ point

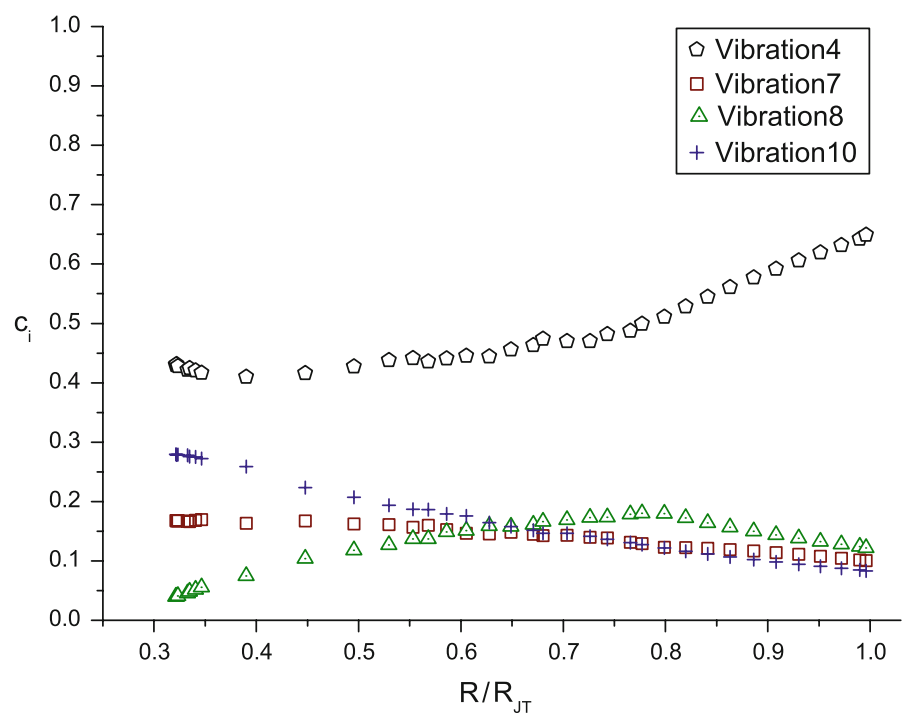

Fig. 10 Changes in the composition of the distortion vector - contribution of the four most important vibrations to the $\mathbf{R}_{\mathrm{JT}}$, given as $c_{\mathrm{k}}$ (linear coefficients in (22) normalized to 1) along the minimal energy (IRC) path

and products, in the fastest way, and due to the fact that in chemical reactions there is bond formation and bond breaking, thus the distortions are much bigger than in the JT cases. This information is obtained from the calculation of the forces at the 
HS point as done by our multimode analysis, Table 7 . Vibration 4 is clearly dominating one, while forces along harder vibrations 7,8 and 10 are smaller and of comparable size. This can be also seen from the values $c_{\mathrm{k}}, E_{\mathrm{k}}$ and IRC calculations. The importance of the vibration 12 (also corresponding to the $e_{2}^{\prime}$ irrep in HS) and 13 (corresponding to the $e_{1}^{\prime}$ irrep in HS) is small but still contributing to the distortion. They contribute each around $1 \%$ to the distortion, and each around $5 \%$ to the $E_{\mathrm{JT}}$, but with not negligible forces at HS point.

\section{Analysis of the Multimode JT Effect at the Stationary Point of Low Symmetry}

As shown in the Sect. 2, the JT theorem predicts a spontaneous distortion of the high symmetry configuration. Group theory allows finding the irreducible representation of the non-totally symmetric vibrations in the HS conformation, which are JT active and remove the degeneracy and lead to a stabilization of the system by lowering the symmetry. The irreducible representations of the active modes, $\Gamma_{\mathrm{HS}}^{v i b}$ are given by the direct product $\Gamma_{\mathrm{HS}}^{\text {elect }} \otimes \Gamma_{\mathrm{HS}}^{\text {elect }} \subset A_{1}+\Gamma_{\mathrm{HS}}^{v i b}$ in $G_{\mathrm{HS}} . G_{\mathrm{LS}}$, the point group of the minimum energy conformation is defined by the requirement that the irreps of the active modes become totally symmetric upon descent in symmetry and application of the epikernal principle $[23,24,46]$.

JT distortion, $\mathbf{R}_{\mathrm{JT}}$ represents a displacement of the nuclei from the HS conformation to the LS energy minimum on the $3 N-6$ dimensional potential energy surface. The minimum is localized by energy minimization constraining the structure to $G_{\mathrm{LS}}$, using well developed algorithms as implemented in standard computational chemistry program packages. The difference between the HS cusp and the LS conformation of minimal energy defines the $\mathbf{R}_{\mathrm{JT}}$. The path from the cusp to the minimum conformation is a reaction coordinate. This has been often overlooked. Therefore, the symmetry rules developed by Bader [9-11] and Pearson [64, 65] can be applied. The JT distortion is defined by the symmetry of the electronic states, as pointed out above and represents a totally symmetric reaction coordinate in $G_{\mathrm{LS}}$. Any displacement on the potential energy surface, also $\mathbf{R}_{\mathrm{JT}}$, has to be totally symmetric and consequently a superposition of the totally symmetric normal coordinates. The number of the later is $N_{a_{1}}$, in general smaller than $3 N-6$.

Within the harmonic approximation, $3 N-6$ dimensional potential energy surface has a simple mathematical form. Because the displacement of the nuclei must be totally symmetric in the $G_{\mathrm{LS}}$, the potential energy surface is defined as a superposition of $N_{a_{1}} \leq 3 N-6$ totally symmetric orthogonal oscillators in LS. In other words the JT distortion is given as a linear combination of displacements along all totally symmetric normal modes in the LS minimum energy conformation. Using this approach it is possible to estimate the contribution of the different normal modes to the $\mathbf{R}_{\mathrm{JT}}$ in a complex system. $N_{a_{1}}$ is in general larger than the number of JT active, $\gamma_{\mathrm{JT}}$, vibrations, which spans $\Gamma_{\mathrm{HS}}^{v i b}$. Because they are of the same symmetry they contribute all to the JT distortion. Especially the $a_{1}$ modes in $G_{\mathrm{HS}}$ mix into 
the $\gamma_{\mathrm{JT}}$ vibrations, because they never change upon descent in symmetry and they are always present in the direct product $\Gamma_{\mathrm{HS}}^{\text {elect }} \otimes \Gamma_{\mathrm{HS}}^{\text {elect }}$. An example is the $E \otimes e$ problem in $T_{d}$ point group discussed for $\mathrm{VCl}_{4}$ in Sect. 4.1. In this case only one component of the angle deformation of $e$ symmetry is $\mathrm{JT}$ active. It changes to $a_{1}$ in $D_{2 d}$. In the lower symmetry it might mix with the $a_{1}$ stretch vibration which is not JT active in $T_{d}$. In many situations other irreps, which are not JT active in HS become totally symmetric upon descent in symmetry, and therefore contribute also to the JT distortion. This is found in the case of the JT $D_{5 h} \rightarrow C_{2 v}$ distortion ${ }^{5}$ already discussed for $\mathrm{C}_{5} \mathrm{H}_{5}$ radical and $\mathrm{CoCp}_{2}$, Sects. 4.2 and 4.3. The normal coordinates that are basis of the $e_{2}^{\prime}, e_{1}^{\prime}$ and $a_{1}^{\prime}$ irreducible representations in $D_{5 h}$ become $a_{1}$ in $C_{2 v} . e_{2}^{\prime} \mathrm{s}$ are JT active in first order, while $e_{1}^{\prime}$ are active in second order.

The choice of the LS geometry as the reference point is in contrast to the usual treatment of the JT effect. This point corresponds to a energy minimum and has the property that the Hessian of the energy is positive semi-definite ${ }^{6}$ and thus can be used to obtain the harmonic vibrational modes without any complications. As already pointed, the totally symmetric subset of vibrations is used to represent potential energy surface of the JT distortion in a harmonic approximation. The HS point in contrary is a cusp on the potential energy surface, the gradientsfirst derivatives of energy over nuclear displacements, are discontinuous and not zero, hence this point is inappropriate for a normal coordinate analysis. Conventional quantum chemistry program packages do not allow to use other points than stationary ones as a reference point in the frequency calculations. Thus, frequency calculations in the HS point will need the implementation of special algorithms into the conventional quantum chemistry packages, e.g. ADF. Of course one can use the results of the normal coordinate analysis of a similar JT-nonactive molecule (e.g. with one electron more or less), but this is an unnecessary approximation. Such a calculation, however, yields no gradients along the JT active modes, which are the essential ingredient of the JT distortions, the force, which drives the molecule out of the high symmetry conformation. In the HS point $a_{1}$ and $\gamma_{\mathrm{JT}}$ normal coordinates do not interact, which is not the case in the LS point, as they are then all of the same symmetry. This allows us to obtain different contribution from the simple linear equation, (29). Furthermore, it is always possible to correlate normal modes of LS to HS ones, thus having connection to the usual treatment based on perturbation theory in HS.

Based on this consideration it is straight forward to analyse the multimode problem using generalized displacement coordinates, $q_{\mathrm{k}}(\mathrm{k}=1 \ldots 3 N)$, around the low symmetry (LS) energy minimum as a origin $\left(q_{\mathrm{LSk}}=0, \mathrm{k}=1 \ldots 3 N\right)$ :

$$
q_{1}=\sqrt{m_{1}} \Delta x_{1}, \quad q_{2}=\sqrt{m_{1}} \Delta y_{1}, \quad \ldots, \quad q_{3 N}=\sqrt{m_{N}} \Delta z_{N}
$$

\footnotetext{
${ }^{5}$ This can be applied of course also for the $D_{5 d}$ and $D_{5}$ point groups.

${ }^{6}$ The square matrix of second-order partial derivatives of a potential energy over the nuclear displacements, Hessian, $\mathbb{H}$, is positive semi-definite if $\mathbf{Q}^{T} \mathbb{H} \mathbf{Q} \geq 0$ for any arbitrary vector $\mathbf{Q}$.
} 
$\Delta x_{\mathrm{n}}, \Delta y_{\mathrm{n}}, \Delta z_{\mathrm{n}}$ are Cartesian displacements from the origin, and $m_{\mathrm{n}}$ are masses of atoms. Every point $\mathbf{X}$ in our conformational space can be represented using this generalized coordinates relative to the origin, by a $3 N$ dimensional vector $\mathbf{R}_{\mathrm{X}}$ :

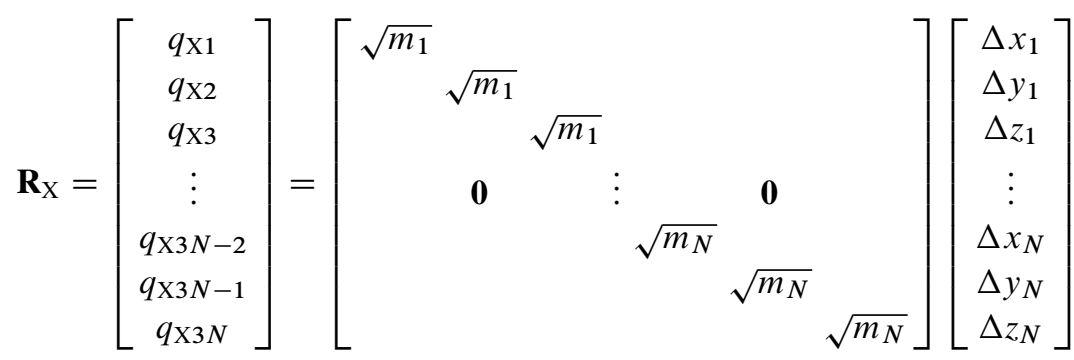

$$
\mathbf{R}_{\mathrm{X}}=\mathbb{M}^{1 / 2} \Delta \mathbf{r}_{\mathrm{X}}
$$

The HS point is given by the vector $\mathbf{R}_{\mathrm{HS}}$ :

$$
\mathbf{R}_{\mathrm{HS}}=\left[\begin{array}{c}
q_{\mathrm{HS} 1} \\
\vdots \\
q_{\mathrm{HS} 3 N}
\end{array}\right]
$$

Consider $q_{\mathrm{HS} 1}, q_{\mathrm{HS} 2}, \ldots, q_{\mathrm{HS} 3 N}$. This is equivalent to say that the JT distortion is equal to $\mathbf{R}_{\mathrm{HS}}, \mathbf{R}_{\mathrm{JT}}=\mathbf{R}_{\mathrm{HS}}$, with elements $q_{\mathrm{HSk}}$.

As the result of the DFT frequency calculations in LS, we have $N_{a_{1}}$ totally symmetric normal coordinates $\mathbf{Q}_{\mathrm{k}}\left(\mathrm{k}=1 \ldots N_{a_{1}}\right)$, which are the eigenvectors of the Hessian. The corresponding eigenvalues are $\lambda_{\mathrm{k}}=\left(2 \pi v_{\mathrm{k}}\right)^{2}, v_{\mathrm{k}}$ is a frequency of a normal mode which is connected to the wave numbers, $\tilde{v}_{\mathrm{k}}$, that are usually used, by a simple relation $v_{\mathrm{k}}=\tilde{v}_{\mathrm{k}} c$, where $c$ is the speed of light. The normal modes are displacement vectors in generalized displacement coordinates, i.e.:

$$
\mathbf{Q}_{\mathrm{k}}=\left[\begin{array}{c}
\Delta q_{\mathrm{k} 1} \\
\vdots \\
\Delta q_{\mathrm{k} 3 N}
\end{array}\right]
$$

Within the harmonic approximation it is possible to express the JT distortion in terms of LS totally symmetric normal coordinates:

$$
\mathbf{R}_{\mathrm{JT}}=\sum_{\mathrm{k}=1}^{N_{a_{1}}} w_{\mathrm{HSk}} \mathbf{Q}_{\mathrm{k}}
$$

In matrix form this yields: 


$$
\begin{gathered}
\mathbf{R}_{\mathrm{JT}}=\left[\begin{array}{c}
q_{\mathrm{HS} 1} \\
\vdots \\
q_{\mathrm{HS} 3 N}
\end{array}\right]=\left[\begin{array}{ccc}
\Delta q_{11} & \ldots & \Delta q_{N_{a_{1}} 1} \\
\vdots & \\
\Delta q_{13 N} & \ldots & \Delta q_{N_{a_{1}} 3 N}
\end{array}\right]\left[\begin{array}{c}
w_{\mathrm{HS} 1} \\
\vdots \\
w_{\mathrm{HS} N_{a_{1}}}
\end{array}\right]=\left[\mathbf{Q}_{1}, \mathbf{Q}_{2}, \ldots, \mathbf{Q}_{N_{a_{1}}}\right]\left[\begin{array}{c}
w_{\mathrm{HS} 1} \\
\vdots \\
w_{\mathrm{HS} N_{a_{1}}}
\end{array}\right] \\
\mathbf{R}_{\mathrm{JT}}=\mathbb{Q} \mathbf{w}_{\mathrm{HS}}
\end{gathered}
$$

This linear problem can be easily solved to get weighting factors $w_{\mathrm{HSk}}$. They represent the contribution of the displacements along the different totally symmetric normal coordinates to the $\mathbf{R}_{\mathrm{JT}}$.

$$
\mathbf{w}_{\mathrm{HS}}=\left(\mathbb{Q}^{T} \mathbb{Q}\right)^{-1} \mathbb{Q}^{T} \mathbf{R}_{\mathrm{JT}}
$$

The weighting can be normalized to 1 , which is more informative as $c_{\mathrm{k}}$ are giving the information of the percentage contribution of each normal mode to the $\mathbf{R}_{\mathrm{JT}}$ :

$$
c_{\mathrm{k}}=\frac{w_{\mathrm{k}}^{2}}{w_{1}^{2}+w_{2}^{2}+\ldots+w_{N_{a_{1}}}^{2}}
$$

The same treatment is possible for any point $\mathbf{R}_{\mathrm{X}}$ on the potential energy surface:

$$
\mathbf{R}_{\mathrm{X}}=\mathbb{Q} \mathbf{w}_{\mathrm{X}}
$$

Alternatively to the method described in Sect. 2, in this harmonic model, $E_{\mathrm{JT}}$ is expressed as the sum of the energy contributions of the totally symmetric normal modes.

$$
E_{\mathrm{JT}}=\sum_{\mathrm{k}=1}^{N_{a_{1}}} E_{\mathrm{k}}=\frac{1}{2} \sum_{\mathrm{k}=1}^{N_{a_{1}}} w_{\mathrm{HSk}}^{2} \mathbf{Q}_{\mathrm{k}}^{2} \lambda_{\mathrm{k}}
$$

Thus each normal mode contributes the energy $E_{\mathrm{k}}$ to the JT stabilisation.

$$
E_{\mathrm{k}}=2 \pi^{2} w_{\mathrm{HSk}}^{2} v_{\mathrm{k}}^{2}\left|\mathbf{Q}_{\mathrm{k}}\right|^{2}
$$

Similarly one can get the potential gradient along each normal coordinate at any point $\mathbf{R}_{\mathrm{X}}$, which is the force, $\mathbf{F}_{\mathrm{Xk}}$, which drives the nuclei along each coordinate to the minimum. $\mathbf{F}_{\mathrm{Xk}}$ is defined as derivative of energy over Cartesian coordinates which yields (38).

$$
\mathbf{F}_{\mathrm{Xk}}=w_{\mathrm{Xk}} \lambda_{\mathrm{k}} \mathbb{M}^{1 / 2} \mathbf{Q}_{\mathrm{k}}
$$

In the HS point this will lead information which normal mode has the steepest descent indicating the main driving force for the JT distortion from the HS to the LS. The total distortion force at a given point is given as the sum of the individual forces, which allows determination of the minimal energy distortion path.

$$
\mathbf{F}_{\mathrm{X}}=\sum_{\mathrm{k}} \mathbf{F}_{\mathrm{Xk}}
$$


As a conclusion, the simple analytical form of the potential energy surface allows to calculate the minimal energy path, step by step from HS to the LS energy minimum. It is obvious that along the path the contributions of the different modes will change. At HS only JT active modes contribute. After the first step the symmetry is lowered and the other modes as mentioned will mix in. This allows getting very detailed picture on the interaction between the deformation of the electron distribution and the displacements of the nuclei.

It must be mentioned that the $3 N-6$ normal coordinates in LS are not identical with the normal coordinates in HS. The correlation between the two systems is however straight forward. There is a unitary transformation between the normal coordinates of HS and LS points:

$$
\left[\mathbf{Q}_{1 \mathrm{HS}}, \mathbf{Q}_{2 \mathrm{HS}}, \ldots, \mathbf{Q}_{N_{a_{1}} \mathrm{HS}}\right]=\left[\mathbf{Q}_{1}, \mathbf{Q}_{2}, \ldots, \mathbf{Q}_{N_{A_{1}}}\right]\left[\begin{array}{ccc}
r_{11} & \ldots & r_{1 N_{a_{1}}} \\
\vdots & & \vdots \\
r_{3 N 1} & \ldots & r_{3 N N_{a_{1}}}
\end{array}\right]
$$

The visual inspection can be used for small molecules, but for larger molecules this is impossible. Alternatively, it is possible to use the method developed by Hug [44], for the comparison of nuclear motions of structurally similar fragments of molecules. We applied it for the correlation of the normal modes of the same molecule in different conformations belonging to different point groups. Using the idea of similarity of the two normal coordinates it is possible to correlate the HS and LS normal coordinates quantitatively. Furthermore it allows using as a reference molecule (HS) a similar molecule which is not JT active, e.g. cyclopentadienyl anion or ferrocene, thus bypassing difficulties in obtaining the normal coordinates of the HS cusp. Hug's program allows pictorial representation of the unitary transformation matrix as shown in Fig. 11 for $\mathrm{C}_{5} \mathrm{H}_{5}$, or in matrix form with numerical values of the similarities, as shown in Table 8 for $\mathrm{CoCp}_{2}$. Identical modes have the value of 1 , while orthogonal value 0 . In schematic representation a circle with a diameter equal to the square which contains it means a value of 1 .

\section{Conclusions and Perspectives}

In this paper a new DFT based method for the qualitative and quantitative analysis of the adiabatic potential energy surfaces of JT active molecules is presented. It is shown how DFT can be successfully applied for the calculation of the JT parameters, and thus be a useful tool in understanding the JT effect and related phenomena. The performance of the model has been evaluated for tetrachlorovanadium(IV) $\left(\mathrm{VCl}_{4}\right)$, an example of ideal, single mode problem; cyclopentadienyl radical $\left(\mathrm{C}_{5} \mathrm{H}_{5}\right)$ and bis(cyclopentadienyl)cobalt(II) (cobaltocene, $\mathrm{CoCp}_{2}$ ) as examples of the multimode problems. The JT parameters obtained using DFT are in excellent agreement with 
Fig. 11 Representation of the similarities of the vibrations in cyclopentadienyl anion (rows), $D_{5 h}$, and radical (columns), $C_{2 v}$; A circle with a diameter equal to the square which contains it means a value of 1 ; vibrations are ordered by increasing energy

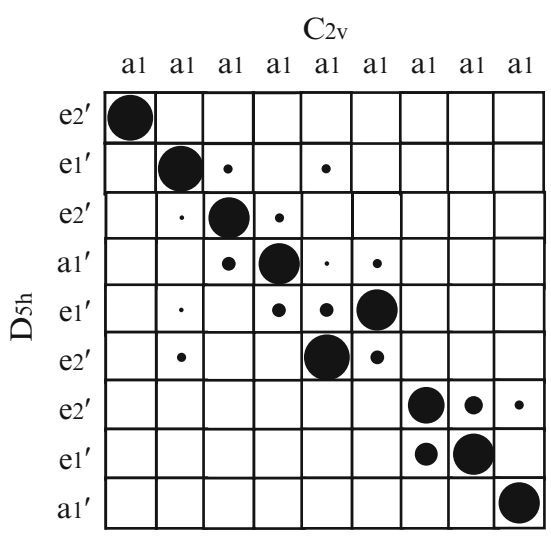

experiment. In addition the importance of the analysis of the multimode JT effect is shown.

JT effect is controlled by the molecular symmetry. Displacements of the nuclei from the JT unstable HS configuration to the LS minimum on the potential energy surface is a totally symmetric reaction coordinate in the $G_{\mathrm{LS}}$ point group. This aspect was often neglected so far. This analogy allows application of the fundamental symmetry rule for reaction coordinates, that it belongs to the totally symmetric irreducible representation of the LS point group of the molecule. Thus, within the harmonic approximation, the distortion can be analysed as the linear combination of totally symmetric normal modes of the LS minimum. This model allows quantifying the contribution of all possible normal modes, their energy contribution to the $E_{\mathrm{JT}}$, the forces at the HS cusp and the detailed distortion path.

There is a sophisticated counterplay between the electronic distortion due to the JT effect, mainly localized on the central metal ion, and the distortion of the ligand conformation in metal complexes. This can lead to a surprising result, e.g. that in $\mathrm{C}_{5} \mathrm{H}_{5}$ the JT distortion does not break the planarity in contrast to the situation in $\mathrm{CoCp}_{2}$. In $\mathrm{C}_{5} \mathrm{H}_{5}$ the ring accepts an en-allyl conformation, whereas in the complex we find a non planar dien conformation. Similar cases are expected in various JT active chelate complexes. Using our method for the multimode analysis presented in this paper, one can get direct insight into the interaction between electronic structure and the nuclear movements. This is of great significance in various fields, not only in the larger JT systems, but also in the systems like spin-crossover compounds, mixed valence compounds, photochemical reactions etc.

\section{Computational Details}

The DFT calculations reported in this work have been carried out using the Amsterdam Density Functional program package, ADF2007.01 [1, 40, 76]. The local density approximation (LDA) characterized by the Vosko- Willk-Nusair (VWN) 


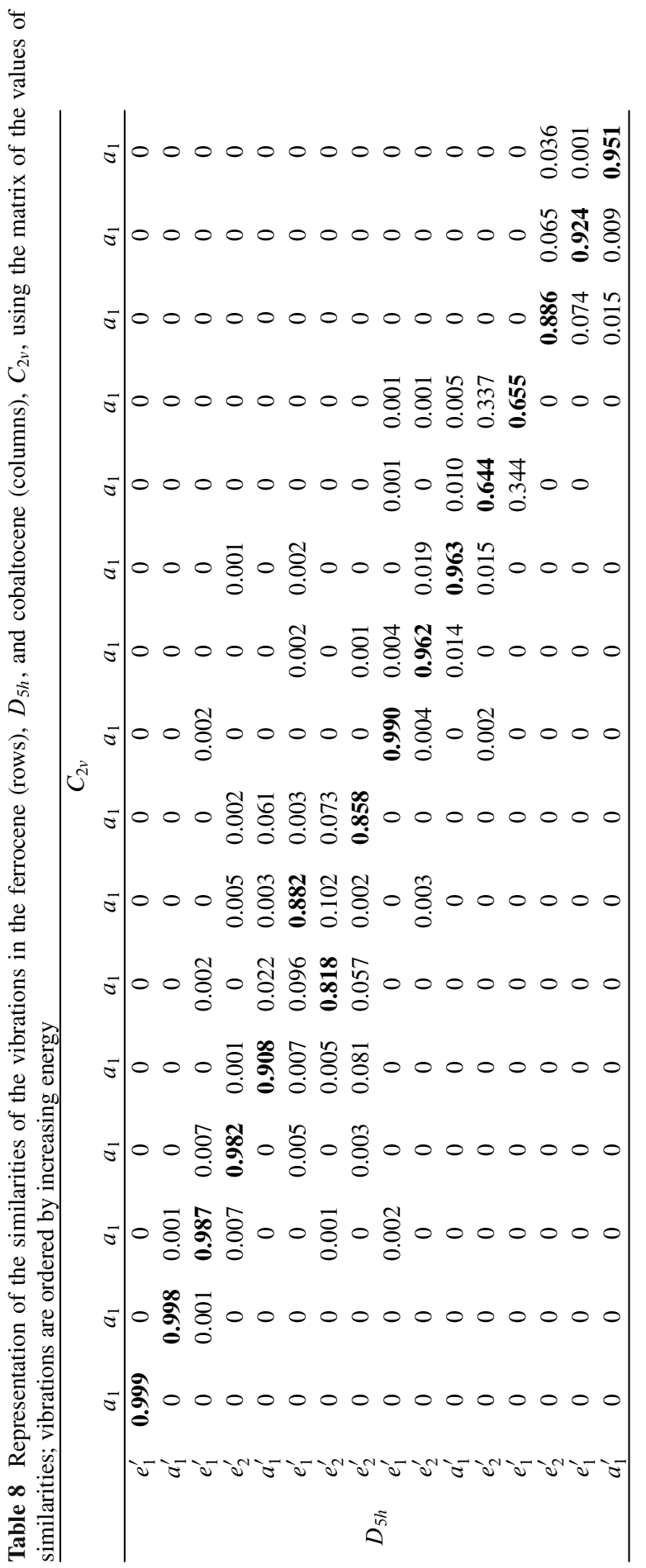


[78] parametrization have been used for the geometry optimizations. Triple zeta (TZP) Slater-type orbital (STO) basis set have been used for all atoms. All calculations were spin-unrestricted with strict criteria for convergence: energy $10^{-4}$ Hartrees; gradients $10^{-4}$ Hartree/ $\AA \AA$; changes in Cartesian coordinates $10^{-4} \AA$; and for numerical integration ten significant digits are used. Analytical harmonic frequencies were calculated $[19,46]$, and were analysed with the aid of PyVib2 1.1 [35]. Vibrations are illustrated using the vibrational energy distribution representation [44]. The different colours indicate the direction of the displacement vector, while the volumes of the spheres are proportional to the contribution made by the individual nuclei to the energy of the vibrational mode. The Intrinsic Reaction Coordinate method [37,38] as implemented in ADF has been used [29,30]. The initial direction of the path is chosen by computing the gradient at the high symmetry configuration. Matlab scripts for the calculation of the coupling coefficients for any point group (Wigner-Eckart theorem) and for the calculation of the weighting factors, $w_{\mathrm{Xk}}$ in (35), can be obtained from authors upon request.

Acknowledgements This work was supported by the Swiss National Science Foundation and the Serbian Ministry of Science (Grant No. 142017G). We thank all the past and present members of our group for their contribution and the valuable discussions.

\section{References}

1. (2007) Adf2007.01. SCM, Theoretical Chemistry, Vrije Universiteit Amsterdam, The Netherlands, http://www.scm.com

2. D.M. Adams, W.S. Fernando, J Chem Soc. Dalton Trans. 222507 (1972)

3. J.H. Ammeter, J.D. Swalen, J. Chem. Phys. 57678 (1972)

4. J.H. Ammeter, L. Zoller, J. Bachmann, P. Baltzer, E. Gamp, R. Bucher, E. Deiss, Helv. Chim. Acta 641063 (1981)

5. B.E. Applegate, T.A. Miller, J. Chem. Phys. 1144855 (2001)

6. B.E. Applegate, A.J. Bezant, T.A. Miller, J. Chem. Phys. 1144869 (2001)

7. P.W. Atkins, M.S. Child, C.S.G. Phillips, Tables for Group Theory (Oxford University Press, Oxford, 1970)

8. F.W. Averill, G.S. Painter, Phys. Rev. B 46(4) 2498 (1992)

9. R.F.W. Bader, Mol. Phys. 3137 (1960)

10. R.F.W. Bader Can. J. Chem. 401164 (1962)

11. R.F.W. Bader, A.D. Bandrauk, J. Chem. Phys. 491666 (1968)

12. M.J. Bearpark, M.A. Robb, N. Yamamoto, Spectrochim. Acta A 55639 (1999)

13. A. Berces, T. Ziegler, Top. Curr. Chem. 18241 (1996)

14. I.B. Bersuker, J Comp. Chem. 18(2) 260 (1997)

15. I.B. Bersuker, The Jahn-Teller Effect (Cambridge University Press, Cambridge, 2006)

16. F.A. Blankenship, R.L. Belford, J. Chem. Phys. 36633 (1962)

17. W.T. Borden, E.R. Davidson, J. Am. Chem. Soc. 1013771 (1979)

18. M. Born, R. Oppenheimer, Ann. Phys. 84457 (1927)

19. A. Brces, R.M. Dickson, L. Fan, H. Jacobsen, D. Swerhone, T. Ziegler, Comput. Phys. Commun. 100247 (1997)

20. J. Brunvoll, S.J. Cyvin, L. Schäfer J. Organomet. Chem. 11459 (1971).

21. R. Bruyndockx, C. Daul, P.T. Manoharan, E. Deiss, Inorg. Chem. 364251 (1997)

22. R. Bucher, Esr-untersuchungen an jahn-teller-aktiven sandwitchkomplexen. $\mathrm{PhD}$ thesis, ETH Zuerich, 1977 
23. A. Ceulemans, L.G. Venquickenborne, Struct. Bonding 71125 (1989)

24. A. Ceulemans, D. Beyens, L.G. Venquickenborne, J. Am. Chem. Soc. 1065824 (1984)

25. M.S. Child, H.C. Longuet-Higgins, Proc. R. Soc. Lond. Ser A 254(1041) 259 (1961)

26. C. Cunha, S. Canuto, J. Mol. Struct: THEOCHEM 46473 (1999)

27. C. Daul, Int. J. Quant. Chem. 52867 (1994)

28. C.A. Daul, K. Doclo, C.A. Stückl Recent Advances in Density Functional Methods, Part II (World Scientific Company, Singapore, 1997), chap On the Calculation of Multiplets

29. L. Deng, T. Ziegler, Int. J. Quant. Chem. 52731 (1994)

30. L. Deng, T. Ziegler, L. Fan, J. Chem. Phys. 993823 (1993)

31. R.M. Dreizler, E.K.U. Gross, Density Functional Theory, an Aproach to Quantum Many-Body Problem (Springer, Berlin, 1990)

32. B.I. Dunlap, W.N. Mei, J. Chem. Phys. 784997 (1983)

33. C. Eckart, Rev. Mod. Phys. 2305 (1930)

34. H. Eicher, Phys. Rev. A 401637 (1989)

35. M. Fedorovsky, Pyvib2, a program for analyzing vibrational motion and vibrational spectra. http://pyvib2.sourceforge.net, 2007

36. M. Filatov, S. Shaik, Chem. Phys. Lett. 304429 (1999)

37. K. Fukui, J. Phys. Chem. 744161 (1970)

38. K. Fukui, Acc. Chem. Res. 14363 (1981)

39. P. Garcia-Fernandez, I.B. Bersuker, J.A. Aramburu, M.T. Barriuso, M. Moreno, Phys. Rev. B 71 184 117-1-10 (2005)

40. C.F. Guerra, J.G. Snijders, G. te Velde, E.J. Baerends, Theor. Chem. Acc. 99391 (1998)

41. G. Herzberg, Moleular Spectra and Molecular Structure III. Electronic Spectra and Electronic Structure of Polyatomic Molecules (Van Norstrand Reinhold Company, Princeton, 1966)

42. G. Herzberg, E. Teller, Z. Phys. Chem. B 21410 (1933)

43. W.D. Hobey, A.D. McLachlan, J. Chem. Phys. 331965 (1960)

44. W. Hug, M. Fedorovsky, Theor. Chem. Acc. 119113 (2008)

45. T. Ichino, S.W. Wren, K.M. Vogelhuber, A.J. Gianola, W.C. Lineberger, J.F. Stanton J. Chem. Phys. 129084 310-084 319 (2008)

46. H. Jacobsen, A. Brces, D. Swerhone, T. Ziegler, Comput. Phys. Commun. 100263 (1997)

47. H.A. Jahn, E. Teller, Proc R Soc Lond. Ser A 161220 (1937)

48. R.B. Johannesen, G.A. Candela, T. Tsang, J. Chem. Phys. 485544 (1968)

49. I.G. Kaplan, J. Mol. Struct. 83839 (2007)

50. J.H. Kiefer, R.S. Tranter, H. Wang, A.F. Wagner, Int. J. Chem. Kin. 33834 (2001)

51. W. Koch, M.C. Holthausen, A Chemis's Guide to Density Functional Theory (Willey-VCH, New York, 2001)

52. E. König, R. Schnakig, S. Kremer, Chem. Phys. 27331 (1978)

53. M. Levy, Phys. Rev. A 261200 (1982)

54. A.D. Liehr, Z. Phys. Chem. 9338 (1956)

55. A.D. Liehr, J. Phys. Chem. 67389 (1963)

56. H.C. Longuet-Higgins, Proc. R. Soc. Lond. Ser. A 344(1637) 147 (1975)

57. H.C. Longuet-Higgins, U. Öpik, M.H.L. Pryce, R.A. Sack, Proc. R. Soc. Lond. Ser. A 244(1236) 1 (1958)

58. N. Matsuzawa, J. Seto, D. Dixon, J. Phys. Chem. A 1019391 (1997)

59. R. Meyer, F. Grof, T. Ha, H.H. Gunthard, Chem. Phys. Lett. 6665 (1979)

60. W. Moffitt, A.D. Liehr, Phys. Rev. 106(6) 1195 (1957)

61. Y. Morino, H. Uehara, J. Chem. Phys. 454543 (1966)

62. U. Öpik, M.H.L. Pryce, Proc. R. Soc. Lond. Ser. A 238(1215) 425 (1957)

63. R.G. Parr, W. Yang, Density-Functional Theory of Atoms and Molecules (Oxford University Press, Oxford, 1989)

64. R.G. Pearson, J. Am. Chem. Soc. 91(18) 4947 (1969)

65. R.G. Pearson, Symmetry Rules for Chemical Reactions (Willey-Interscience, New York, 1976)

66. R. Renner, Z. Phys. A 92172 (1934)

67. T. Satoa, K. Tanaka, K. Tokunaga, J. Chem. Phys. 124124 (2006)

68. L. Schäfer, J. Brunvoll, S.J. Cyvin, J. Mol. Struct. 11459 (1972) 
69. P.R.T. Schipper, O.V. Gritsenko, E.J. Baerends, Theor. Chim. Acc. 99329 (1998)

70. J. Simons, Energetic Principles of Chemical Reactions (Jones and Bartlett, Boston, 1983)

71. J.C. Slater, J.B. Mann, T.M. Wilson, J.H. Wood, Phys. Rev. 184672 (1969)

72. L.C. Snyder, J. Chem. Phys. 33619 (1960)

73. J.F. Stanton, J. Chem. Phys. 11510382 (2001)

74. A. Stebler, A. Furrer, J.H. Ammeter, Inorg. Chem. 233493 (1984)

75. M. Swart, Inorg. Chim. Acta 360179 (2007)

76. G. te Velde, F.M. Bickelhaupt, S.J.A van Gisbergen, C.F. Guerra, E.J. Baerends, J.G. Snijders, T. Ziegler, J. Comput. Chem. 22931 (2001)

77. J.H. van Vleck, J. Chem. Phys. 772 (1939)

78. S. Vosko, L. Wilk, M. Nusair, Can. J. Phys. 581200 (1980)

79. S.G. Wang, H.E. Shwarz,J. Chem. Phys. 1054641 (1996)

80. J. Weber, A. Goursot, E. Pénigault, J.H. Ammeter, J. Bachmann, J. Am. Chem. Soc. 1041491

81. E.P. Wigner,(1930) Gruppentheorie (Vieweg, Braunschweig, 1930)

82. Z.F. Xu, Y. Xie, W.L. Feng, H.F. SchaeferIII, J. Phys. Chem. A 1072176 (1997)

83. Y. Zhang, W. Yang, J. Chem. Phys. 1092604 (1998)

84. T. Ziegler, A. Rauk, E.J. Baerends, Theor. Chim. Acta 43261 (1977)

85. S. Zilberg, Y. Haas, J. Am. Chem. Soc. 12410683 (2002)

86. M. Zlatar, C.W. Schläpfer, E.P. Fowe, C. Daul, Pure Appl. Chem. 811397 (2009) 